Polarimetric synthetic-aperture inversion for extended targets in clutter

This content has been downloaded from IOPscience. Please scroll down to see the full text. 2013 Inverse Problems 29054003

(http://iopscience.iop.org/0266-5611/29/5/054003)

View the table of contents for this issue, or go to the journal homepage for more

Download details:

IP Address: 128.113.62.199

This content was downloaded on 18/11/2013 at 18:36

Please note that terms and conditions apply. 


\title{
Polarimetric synthetic-aperture inversion for extended targets in clutter
}

\author{
Kaitlyn Voccola ${ }^{1,2,3}$, Margaret Cheney ${ }^{3,4}$ and Birsen Yazici ${ }^{5}$ \\ ${ }^{1}$ Sensors Directorate, Air Force Research Laboratory, Dayton, OH 45433-7318, USA \\ 2 Department of Mathematical Sciences, Rensselaer Polytechnic Institute, Troy, NY 12180-3509, \\ USA \\ ${ }^{3}$ Department of Mathematics and Department of Electrical and Computer Engineering, Colorado \\ State University, Fort Collins, CO 80523, USA \\ ${ }^{4}$ Department of Applied Mathematics, Naval Postgraduate School, Monterey, CA 93943-5216, \\ USA \\ ${ }^{5}$ Department of Electrical, Computer and System Engineering, Rensselaer Polytechnic Institute, \\ Troy, NY 12180-3590, USA \\ E-mail: kvoccola@gmail.com
}

Received 16 May 2012, in final form 31 January 2013

Published 18 April 2013

Online at stacks.iop.org/IP/29/054003

\begin{abstract}
This paper presents an analytic inversion method for a polarimetric syntheticaperture radar (SAR) in the case of an extended target embedded in clutter. The measurements are also contaminated by thermal noise. We use microlocal analysis in a statistical setting to develop a filtered-backprojectiontype reconstruction method. The inversion method accommodates arbitrary waveforms and arbitrary flight paths. We model the antennas and scatterers as dipoles; scatterers are thus characterized by a spatially varying scattering matrix. We include directional scattering assumptions to distinguish a curvelike extended target from clutter, which is assumed to scatter isotropically. For the inversion we choose the backprojection filter which minimizes the meansquare error between the reconstructed image and the actual target scattering matrix. Our work differs from standard polarimetric SAR imaging in that we do not perform channel-by-channel image reconstruction. We find that it is preferable to use a coupled reconstruction scheme in which we use all sets of collected data to form each element of the scattering matrix. We show in our numerical experiments that the coupled reconstruction not only minimizes the mean-square error but also improves the image target-to-clutter ratio in certain scenarios.
\end{abstract}

(Some figures may appear in colour only in the online journal)

\section{Introduction}

In synthetic-aperture radar (SAR) imaging [27], a moving antenna illuminates a scene, which here is assumed to consist of both scatterers of interest (the target) and scatterers that are not 
of interest (clutter). The transmitted electromagnetic waves (generally microwaves) scatter from the scene and are typically measured at the same (or a nearby) moving antenna; the measurements are then used to form an image of the scene.

This paper focuses on the extension of a hybrid SAR image-formation technique that uses both analytical and statistical theory in the framework of backprojection. The technique was previously developed for the scalar case in [42], which found that incorporating the statistics of the scene into the imaging algorithm leads to clutter mitigation and also minimizes the effect of noise on the image. We extend these results for a full vector treatment of the transmission and scattering of the electromagnetic waves. That is, we consider the case when a fully polarimetric radar system is used. We present a technique that gives quantifiable improvements in image quality, namely reduced mean-square error (MSE) and improved image target-to-clutter ratio, which is commonly referred to as the signal-to-clutter ratio (SCR).

We note that most work in polarimetry has been based on estimation-theoretic approaches $[11,12,8,13,24,14,28,4,29,26,18,31,3,19,34,40]$. It is typically assumed that one can reconstruct each element of the target scattering matrix from the corresponding data set. This assumption stems from the idea that the sensor's orientation, and therefore polarization, is fixed with respect to the scene of interest [5]. Therefore, standard imaging schemes are applied to each set of data separately. However, this assumption holds only when the antennas in question have a narrow azimuthal beamwidth. One situation where this assumption does not hold is a SAR system operating at lower frequency bands (i.e. L-band). In these systems, the antenna polarization properties vary with azimuthal look angle. In [5], a subaperture data processing approach is implemented to handle scenarios where the fixed orientation assumption does not hold. SAR images are decomposed into subaperture data sets to visualize the different responses under different azimuthal look angles. One drawback of using subaperture data sets is a reduction in resolution.

In this paper, we avoid the narrow-beam assumption. For wide-beam synthetic-aperture radars, we find that it is optimal (in the mean-square sense) to use a coupled image formation technique, which uses every data set to reconstruct each element of the target scattering matrix. This coupled reconstruction increases the computation time of the imaging algorithm, but improves MSE and image SCR as stated above.

We stress that our work differs significantly from most of the studies in polarimetry. We do not assume that we already have an accurate estimate of the scattering matrix and then attempt to perform target decomposition as most researchers do in polarimetry [11, 19, 20]. We instead begin with Maxwell's equations and derive a simplified, physics-based forward scattering model (see below). For this model, we develop a novel reconstruction, or estimation, of the scattering matrix.

In developing this polarimetric imaging scheme, we consider specifically extended targets embedded in clutter. By 'extended', we mean one-dimensional targets such as fences and power lines. Such targets not only affect the polarization of the incident field, but they also scatter electromagnetic waves anisotropically.

Anisotropic scattering has been studied previously by a number of different researchers $[1,6,16]$. For example in [1], the radar data are broken up into data received from different sets of observation angles, or different intervals of the bandwidth. This does help to characterize the different returns from different angles, but it leads to a reduction in resolution because the data are restricted to smaller apertures or smaller bandwidths. In contrast, our approach is to incorporate the anisotropy in the scattering model.

In order to incorporate both anisotropy and changes in polarization, we assume that all scatterers in the scene are made up of non-interacting scattering elements consisting of short dipoles. The dipole scatterers have an orientation and a location, and they display anisotropic 
scattering behavior. This dipole scattering model was previously considered in [16]. Our work differs from [16] in that we make certain simplifying assumptions that allow us to obtain an analytic expression for the image. The work [16], on the other hand, focuses on a purely numerical approach.

We emphasize that, as usual for analytic models used for reconstruction or imaging, our scattering model omits many physical effects. The key is always to develop a scattering model that incorporates as much physics as possible, but is still simple enough to be inverted. We feel that our model is an improvement over most existing models, which use non-interacting scattering elements that are point-like. Of course, the ultimate test of the utility of this model is in its success with real data, which is a study we leave for the future.

The remainder of the paper is organized as follows. In section 2, we give some preliminary background information on polarimetry and the typical scattering model used in polarimetric radar. In addition, we derive our dipole SAR forward model and highlight the differences between our model and the standard models. In section 3, we discuss our image-formation approach, in particular the method of backprojection, and define our imaging operator. In this section, we derive the optimal filter (in the mean-square sense) for the case when the target and clutter are statistically independent. Finally, in section 4, we include numerical simulations in which we model the data received at the radar. We also include results of using our coupled imaging scheme and compare with standard polarimetric SAR imaging.

We use the convention that vectors appear in bold font (e.g. $\boldsymbol{x}$ ), and matrices are underlined (e.g. $\underline{A})$.

\section{Forward model}

\subsection{Polarimetry background}

It is well known [10, 17, 21, 36-38] that in free space, Maxwell's equations for a time-harmonic field simplify to the Helmholtz equation

$$
\left(\nabla^{2}+k^{2}\right) \boldsymbol{E}(k, \boldsymbol{x})=\mathbf{0}
$$

whose simplest solution is a plane wave of the form

$$
\boldsymbol{E}(\boldsymbol{x})=\boldsymbol{E} \mathrm{e}^{\mathrm{i} k \cdot \boldsymbol{x}}
$$

where $\boldsymbol{E}$ is a constant-amplitude field vector and $\boldsymbol{k}=k \hat{\boldsymbol{k}} \in \mathbb{R}^{3}$ where $k=\omega / c$ with $c$ being the speed of light, $\omega$ being angular frequency and $\hat{\boldsymbol{k}}$ being the direction of propagation. We define a right-handed coordinate system, denoted $(\hat{\boldsymbol{h}}, \hat{\boldsymbol{v}}, \hat{\boldsymbol{k}})$, in which $\hat{\boldsymbol{h}}$ and $\hat{\boldsymbol{v}}$ span the plane perpendicular to $\hat{\boldsymbol{k}}$. From Maxwell's equations we infer that $\boldsymbol{E}$ lies in this plane and therefore may be written as the linear combination

$$
\boldsymbol{E}=E_{h} \hat{\boldsymbol{h}}+E_{v} \hat{\boldsymbol{v}} .
$$

In the typical polarimetric scattering scenario, the transmitting antenna is assumed to transmit a monochromatic plane wave with the propagation direction $\hat{\boldsymbol{k}}_{i}$. This field $\boldsymbol{E}^{i}$ can be written as

$$
\boldsymbol{E}^{i}=E_{h}^{i} \hat{\boldsymbol{h}}_{i}+E_{v}^{i} \hat{\boldsymbol{v}}_{i}
$$

where the basis vectors $\hat{\boldsymbol{h}}_{i}$ and $\hat{\boldsymbol{v}}_{i}$ define the plane perpendicular to $\hat{\boldsymbol{k}}_{i}$. When this wave interacts with a scatterer, the polarization state and/or degree of polarization may change. We assume that the wave which scatters from the target is received at the antenna which lies in the direction $\hat{\boldsymbol{k}}_{s}$, in the far field of the scatterer. We therefore express the scattered field vector in terms of the basis vectors defining the plane perpendicular to $\hat{\boldsymbol{k}}_{s}$ :

$$
\boldsymbol{E}^{s}=E_{h}^{s} \hat{\boldsymbol{h}}_{s}+E_{v}^{s} \hat{\boldsymbol{v}}_{s} .
$$


Note that the right-handed coordinate system $\left(\hat{\boldsymbol{h}}_{s}, \hat{\boldsymbol{v}}_{s}, \hat{\boldsymbol{k}}_{s}\right)$ is not the same as the system $\left(\hat{\boldsymbol{h}}_{i}, \hat{\boldsymbol{v}}_{i}, \hat{\boldsymbol{k}}_{i}\right)$. The mapping from $\boldsymbol{E}^{i}$ to $\boldsymbol{E}^{s}$ can be thought of as a transformation performed by the scatterer. We describe this transformation mathematically as

$$
\boldsymbol{E}^{s}=\underline{S} \boldsymbol{E}^{i}=\left(\begin{array}{ll}
S_{h h} & S_{h v} \\
S_{v h} & S_{v v}
\end{array}\right) \boldsymbol{E}^{i}
$$

where $\underline{S}$ is known as the scattering matrix for the scatterer. This will be incorporated into the quantity we reconstruct later when we discuss polarimetric imaging. Note that $\underline{S}$ depends on the transmitted frequency, the scatterer, the scattering geometry and the basis we use to describe the waves. In polarimetric SAR, we typically attempt to measure the scattering matrix by transmitting two orthogonal polarizations, one after the other, and then receiving the scattered waves in the same two orthogonal polarizations.

\subsection{Dipole SAR forward model}

To derive the dipole SAR model, we assume our SAR system is made up of two dipole antennas, $a$ and $b$, which travel along paths $\boldsymbol{\gamma}_{a}(s)$ and $\boldsymbol{\gamma}_{b}(s)$. Here $s$ parametrizes the antenna trajectories and is known as the slow time parameter and corresponds to the time scale on which the antenna moves; in contrast, $t$ is known as the fast time parameter and corresponds to the time scale on which light propagates. We assume that dipole $a$ transmits the waveform $p_{a}(t)$, and the scattered field is received on both $a$ and $b$. Similarly, dipole $b$ transmits the waveform $p_{b}(t)$, and the scattered field is received on both $a$ and $b$. We denote the Fourier transforms of the waveforms by $P_{a}$ and $P_{b}$. We also assume that the dipoles have directions $\widehat{\boldsymbol{e}}_{a}$ and $\widehat{\boldsymbol{e}}_{b}$, respectively.

We model scatterers as a collection of dipoles located at various pixels and with various orientations. We denote by $\widehat{\boldsymbol{e}}_{T}(\boldsymbol{x})$ the orientation of the target dipole at location $\boldsymbol{x}$ and by $\widehat{\boldsymbol{e}}_{C}(\boldsymbol{x})$ the orientation of the clutter dipole. We also assume the measurements are corrupted by noise $n$. Therefore our forward model in the frequency domain is of the form

$$
D_{i, j}(k, s)=\left(\mathcal{F}^{T}[\boldsymbol{T}]\right)_{i, j}(k, s)+\left(\mathcal{F}^{C}[\boldsymbol{C}]\right)_{i, j}(k, s)+n_{i, j}(k, s),
$$

where $i=a, b$ and $j=a, b$. We call $D_{i, j}$ the set of the data collected when we transmit on the $i$ th antenna and receive on the $j$ th antenna. Here, $\boldsymbol{T}$ and $\boldsymbol{C}$ are the vector functions that describe the target and clutter, respectively, to be described in more detail below, and $n_{i, j}$ is the noise that corrupts the measurements when we transmit on $i$ and receive on $j$. The 'forward' operators $\mathcal{F}^{T}$ and $\mathcal{F}^{C}$ map the target and clutter vector fields to the corresponding received data. These operators are derived below, directly from solutions of Maxwell's equations. We now go into more detail to describe (a) the radiation from the transmitting dipole antenna, (b) the scattering from the dipoles that make up the target and clutter and (c) the reception on the receiving dipole antenna.

2.2.1. Radiation from the transmitting dipole antenna. In the frequency domain, the far-field electric field due to a radiating dipole of length $a$, located at position $\gamma_{a}(s)$ and pointing in the direction $\widehat{\boldsymbol{e}}_{a}$, is $[21,23,7,25]$

$$
\begin{gathered}
\qquad \boldsymbol{E}_{a}(k, \boldsymbol{x})=\widehat{\boldsymbol{R}}_{\boldsymbol{x}, s}^{a} \times\left(\widehat{\boldsymbol{R}}_{\boldsymbol{x}, s}^{a} \times \widehat{\boldsymbol{e}}_{a}\right) \frac{\mathrm{e}^{\mathrm{i} k R_{\boldsymbol{x}, s}^{a}}}{4 \pi R_{\boldsymbol{x}, s}^{a}} F^{a}\left(k \widehat{\boldsymbol{R}}_{\boldsymbol{x}, s}^{a} \cdot \widehat{\boldsymbol{e}}_{a}\right) P_{a}(k), \\
\text { where } \boldsymbol{R}_{\boldsymbol{x}, s}^{a}=\boldsymbol{x}-\boldsymbol{\gamma}_{a}(s), R_{\boldsymbol{x}, s}^{a}=\left|\boldsymbol{R}_{\boldsymbol{x}, s}^{a}\right| \text { and } \\
F^{a}(k \cos \theta)=a \operatorname{sinc}\left(\frac{k a}{2} \cos \theta\right)
\end{gathered}
$$


is the antenna pattern of the dipole $a$. This expression results from a method-of-potentials solution of Maxwell's equations. For more details on this solution of Maxwell's equations, see [10, 17, 23, 25, 36, 37, 41].

In practice, SAR systems are side-looking: they use antenna beam patterns that illuminate only parts of the scene to one side of the flight path. This is done to avoid the 'left-right' ambiguity, which is a certain image artifact [7]. Without further comment, we will assume that the SAR system has in fact been designed to avoid such artifacts.

2.2.2. Interaction of the field with the scatterer. We assume that every scatterer in the scene is modeled as a dipole located at the position $\boldsymbol{x}$ and pointing in the direction $\widehat{\boldsymbol{e}}_{\mathrm{sc}}(\boldsymbol{x})$. Note we use the subscript and superscript sc to indicate this scatterer may be part of the extended target or a clutter scatterer. Each dipole making up the scatterer acts as a receiving antenna with antenna pattern $F^{\mathrm{sc}}$. We can calculate the current excited on the dipole at the position $\boldsymbol{x}$ and pointing in the direction $\widehat{\boldsymbol{e}}_{\mathrm{sc}}(\boldsymbol{x})$, due to the incident field $\boldsymbol{E}_{a}$. We have

$$
\begin{aligned}
I_{\mathrm{sc}} \propto \widehat{\boldsymbol{e}}_{\mathrm{sc}} \cdot \boldsymbol{E}_{a} F^{\mathrm{sc}} & \left(k \widehat{\boldsymbol{R}}_{\boldsymbol{x}, s}^{a} \cdot \widehat{\boldsymbol{e}}_{\mathrm{sc}}\right) \\
= & \widehat{\boldsymbol{e}}_{\mathrm{sc}} \cdot\left[\widehat{\boldsymbol{R}}_{\boldsymbol{x}, s}^{a} \times\left(\widehat{\boldsymbol{R}}_{\boldsymbol{x}, s}^{a} \times \widehat{\boldsymbol{e}}_{a}\right)\right] F^{a}\left(k \widehat{\boldsymbol{R}}_{\boldsymbol{x}, s}^{a} \cdot \widehat{\boldsymbol{e}}_{a}\right) F^{\mathrm{sc}}\left(k \widehat{\boldsymbol{R}}_{\boldsymbol{x}, s}^{a} \cdot \widehat{\boldsymbol{e}}_{\mathrm{sc}}\right) \frac{\mathrm{e}^{\mathrm{i} k R_{\boldsymbol{x}, s}^{a}}}{4 \pi R_{\boldsymbol{x}, s}^{a}} P_{a}(k) .
\end{aligned}
$$

We assume that the current induced on the dipole radiates again as a dipole antenna, and in this process has strength $\rho(\boldsymbol{x})$ and again antenna pattern $F^{\mathrm{sc}}$.

2.2.3. Reception at the receiving dipole antenna. The measured data are given by the current on the dipole located at the position $\boldsymbol{\gamma}_{b}(s)$ with orientation $\widehat{\boldsymbol{e}}_{b}$. We calculate this as in equation (10). We have

$$
\begin{aligned}
D_{a, b}(k, s) \propto \widehat{\boldsymbol{e}}_{b} \cdot \boldsymbol{E}\left(k, \boldsymbol{\gamma}_{b}(s)\right)= & \int \rho(\boldsymbol{x}) \frac{\mathrm{e}^{\mathrm{i} k\left(R_{x, s}^{a}+R_{\boldsymbol{x}, s}^{b}\right)}}{16 \pi^{2} R_{\boldsymbol{x}, s}^{a} R_{\boldsymbol{x}, s}^{b}} F^{\mathrm{sc}}\left(k \widehat{\boldsymbol{R}}_{\boldsymbol{x}, s}^{a} \cdot \widehat{\boldsymbol{e}}_{\mathrm{sc}}\right) F^{\mathrm{sc}}\left(k \widehat{\boldsymbol{R}}_{\boldsymbol{x}, s}^{b} \cdot \widehat{\boldsymbol{e}}_{\mathrm{sc}}\right) \\
& \times F^{b}\left(k \widehat{\boldsymbol{R}}_{\boldsymbol{x}, s}^{b} \cdot \widehat{\boldsymbol{e}}_{b}\right) F^{a}\left(k \widehat{\boldsymbol{R}}_{\boldsymbol{x}, s}^{a} \cdot \widehat{\boldsymbol{e}}_{a}\right) P_{a}(k) \\
& \times \widehat{\boldsymbol{e}}_{\mathrm{sc}} \cdot\left[\widehat{\boldsymbol{R}}_{\boldsymbol{x}, s}^{a} \times\left(\widehat{\boldsymbol{R}}_{\boldsymbol{x}, s}^{a} \times \widehat{\boldsymbol{e}}_{a}\right)\right] \widehat{\boldsymbol{e}}_{b} \cdot\left[\widehat{\boldsymbol{R}}_{\boldsymbol{x}, s}^{b} \times\left(\widehat{\boldsymbol{R}}_{\boldsymbol{x}, s}^{b} \times \widehat{\boldsymbol{e}}_{\mathrm{sc}}\right)\right] \mathrm{d} \boldsymbol{x},
\end{aligned}
$$

where we have taken the additional step of integrating over all possible ground locations $\boldsymbol{x}$ in the scene. Here, we have also suppressed the dependence of $\widehat{\boldsymbol{e}}_{\mathrm{sc}}$ on $\boldsymbol{x}$ for ease of notation. The two subscripts on the left-hand side of (11) indicate that we transmit on dipole $a$ and receive on dipole $b$.

2.2.4. Vector formulation. We note that the vector expressions on the last line of (11) can be rewritten with the help of the triple product, or BAC-CAB, identity as

$$
\begin{aligned}
\widehat{\boldsymbol{e}}_{\mathrm{sc}} \cdot\left[\widehat{\boldsymbol{R}}_{\boldsymbol{x}, s}^{a} \times\left(\widehat{\boldsymbol{R}}_{\boldsymbol{x}, s}^{a} \times \widehat{\boldsymbol{e}}_{a}\right)\right] \widehat{\boldsymbol{e}}_{b} \cdot\left[\widehat{\boldsymbol{R}}_{\boldsymbol{x}, s}^{b} \times\left(\widehat{\boldsymbol{R}}_{\boldsymbol{x}, s}^{b} \times \widehat{\boldsymbol{e}}_{\mathrm{sc}}\right)\right] \\
=\left[\left(\widehat{\boldsymbol{R}}_{\boldsymbol{x}, s}^{a} \times \widehat{\boldsymbol{e}}_{\mathrm{sc}}\right) \cdot\left(\widehat{\boldsymbol{R}}_{\boldsymbol{x}, s}^{a} \times \widehat{\boldsymbol{e}}_{a}\right)\right]\left[\left(\widehat{\boldsymbol{R}}_{\boldsymbol{x}, s}^{b} \times \widehat{\boldsymbol{e}}_{\mathrm{sc}}\right) \cdot\left(\widehat{\boldsymbol{R}}_{\boldsymbol{x}, s}^{b} \times \widehat{\boldsymbol{e}}_{b}\right)\right] \\
=\left[\widehat{\boldsymbol{R}}_{\boldsymbol{x}, s}^{a} \times\left(\widehat{\boldsymbol{R}}_{\boldsymbol{x}, s}^{a} \times \widehat{\boldsymbol{e}}_{a}\right)\right] \cdot \widehat{\boldsymbol{e}}_{\mathrm{sc}}\left[\widehat{\boldsymbol{R}}_{\boldsymbol{x}, s}^{b} \times\left(\widehat{\boldsymbol{R}}_{\boldsymbol{x}, s}^{b} \times \widehat{\boldsymbol{e}}_{b}\right)\right] \cdot \widehat{\boldsymbol{e}}_{\mathrm{sc}}
\end{aligned}
$$

In addition, we may rewrite (12) using tensor products:

$\left[\widehat{\boldsymbol{R}}_{\boldsymbol{x}, s}^{a} \times\left(\widehat{\boldsymbol{R}}_{\boldsymbol{x}, s}^{a} \times \widehat{\boldsymbol{e}}_{a}\right)\right] \cdot \widehat{\boldsymbol{e}}_{\mathrm{sc}}\left[\widehat{\boldsymbol{R}}_{\boldsymbol{x}, s}^{b} \times\left(\widehat{\boldsymbol{R}}_{\boldsymbol{x}, s}^{b} \times \widehat{\boldsymbol{e}}_{b}\right)\right] \cdot \widehat{\boldsymbol{e}}_{\mathrm{sc}}=\left(\boldsymbol{R}_{a}^{\perp} \otimes \boldsymbol{R}_{b}^{\perp}\right):\left(\widehat{\boldsymbol{e}}_{\mathrm{sc}} \otimes \widehat{\boldsymbol{e}}_{\mathrm{sc}}\right)$,

where $\boldsymbol{R}^{\perp}=\hat{\boldsymbol{R}} \times(\hat{\boldsymbol{R}} \times \hat{\boldsymbol{e}}), \otimes$ is the standard tensor product and : is the double dot product (the matrix analogue to the vector dot product). 


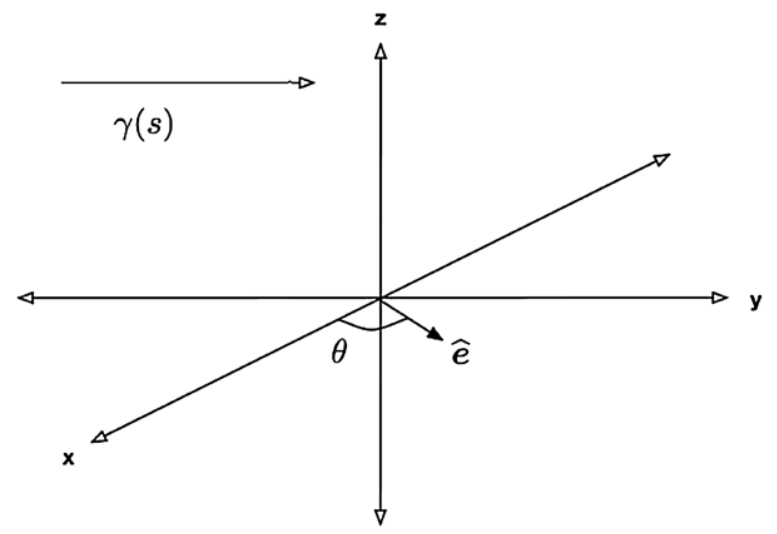

Figure 1. Target-centered coordinate system.

If we receive on both $a$ and $b$, we obtain the data vector

$$
\boldsymbol{D}(k, s)=\left(\begin{array}{c}
D_{a, a} \\
D_{a, b} \\
D_{b, a} \\
D_{b, b}
\end{array}\right)
$$

The data vector $\boldsymbol{D}$ is dependent on a vector of quantities like those in equation (13) but with different transmitting and receiving antenna pairs. This vector may be expressed as the product of a matrix and vector as follows. If $\boldsymbol{R}_{a}^{\perp}=\left(x_{a}, y_{a}, z_{a}\right), \boldsymbol{R}_{b}^{\perp}=\left(x_{b}, y_{b}, z_{b}\right)$ and $\widehat{\boldsymbol{e}}_{\mathrm{sc}}(\boldsymbol{x})=\left[\cos \theta_{\mathrm{sc}}(\boldsymbol{x}), \sin \theta_{\mathrm{sc}}(\boldsymbol{x}), 0\right]$, then

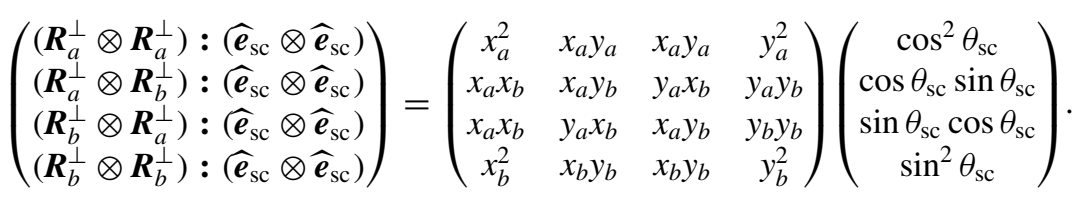

Here, we use a scatterer-centered coordinate system where the angle $\theta_{\mathrm{sc}}$ is defined with respect to the $x$-axis as in figure 1. We again suppress the dependence of $\theta_{\mathrm{sc}}$ on $\boldsymbol{x}$ for ease of notation. Note that the third coordinate of $\boldsymbol{R}^{\perp}$ does not appear in (15) because in the double dot product, the third coordinate is multiplied by the third coordinate of $\widehat{\boldsymbol{e}}_{\mathrm{sc}}$ which is always zero. Also note that we now have expressed our forward model in terms of the quantity

$$
\boldsymbol{S}\left(\theta_{\mathrm{sc}}\right)=\left(\begin{array}{c}
\cos ^{2} \theta_{\mathrm{sc}} \\
\cos \theta_{\mathrm{sc}} \sin \theta_{\mathrm{sc}} \\
\sin \theta_{\mathrm{sc}} \cos \theta_{\mathrm{sc}} \\
\sin ^{2} \theta_{\mathrm{sc}}
\end{array}\right)
$$

which we call the scattering vector, or the vectorized scattering matrix.

Our usage differs from that in the polarimetry literature, where the scattering matrix as in equation (6) for a dipole scatterer [11, 18, 22, 30, 32, 35, 39] is given as the full expression in equation (15). This definition depends on antenna parameters, whereas our model (16) separates the antenna characteristics from that of the scatterer. In particular, the vectors $\boldsymbol{R}_{a}^{\perp}$ and $\boldsymbol{R}_{b}^{\perp}$ may be written with the help of the BAC-CAB identity as

$$
\hat{\boldsymbol{R}} \times(\hat{\boldsymbol{R}} \times \hat{\boldsymbol{e}})=\hat{\boldsymbol{R}}(\hat{\boldsymbol{R}} \cdot \hat{\boldsymbol{e}})-\hat{\boldsymbol{e}}(\hat{\boldsymbol{R}} \cdot \hat{\boldsymbol{R}})=-[\hat{\boldsymbol{e}}-\hat{\boldsymbol{R}}(\hat{\boldsymbol{R}} \cdot \hat{\boldsymbol{e}})]=-P_{\widehat{\boldsymbol{R}}}^{\perp},
$$


where $\hat{\boldsymbol{e}}$ is either $\hat{\boldsymbol{e}}_{a}$ or $\hat{\boldsymbol{e}}_{b}$ and where $P_{\widehat{\boldsymbol{R}}}^{\perp}$ denotes the operator that projects a vector onto the plane perpendicular to the direction of propagation $\widehat{\boldsymbol{R}}$. In other words, we see that (17) projects the antenna orientation $\hat{\boldsymbol{e}}$ onto the plane perpendicular to the direction of propagation. Therefore the vector on the right-hand side of (17) is the same as the transmitted field's state in equation (3). As is commonly done, we make the standard assumption that antenna polarization $\hat{\boldsymbol{e}}$ remains fixed as the antenna moves along its flight path. For systems in which the antenna beam is very narrow, $\widehat{\boldsymbol{R}}$ can be assumed to be constant over the antenna footprint, and consequently the entire expression (15) is typically regarded as the (target plus clutter) scattering vector field. In this paper, we do not assume a narrow antenna beam, and consequently we address the variation of $\widehat{\boldsymbol{R}}$ over the antenna footprint. In our case, it is not appropriate to consider the entire expression (15) to be the scattering vector field. Instead, our (target plus clutter) scattering vector field consists of only the quantity in equation (16). Note that both $\boldsymbol{S}\left(\theta_{\mathrm{sc}}\right)$ and $\rho(\boldsymbol{x})$ are unknown.

We now make the assumption that antennas $a$ and $b$ are collocated, that is, we assume a monostatic system. Therefore, we have the following data expression for any dipole scatterer:

$\boldsymbol{D}(k, s)=\int \mathrm{e}^{2 \mathrm{i} k R_{x, s}}\left(\begin{array}{cccc}\mathcal{A}_{a, a} x_{a}^{2} & \mathcal{A}_{a, a} x_{a} y_{a} & \mathcal{A}_{a, a} x_{a} y_{a} & \mathcal{A}_{a, a} y_{a}^{2} \\ \mathcal{A}_{a, b} x_{a} x_{b} & \mathcal{A}_{a, b} x_{a} y_{b} & \mathcal{A}_{a, b} y_{a} x_{b} & \mathcal{A}_{a, b} y_{a} y_{b} \\ \mathcal{A}_{b, a} x_{a} x_{b} & \mathcal{A}_{b, a} y_{a} x_{b} & \mathcal{A}_{b, a} x_{a} y_{b} & \mathcal{A}_{b, a} y_{b} y_{b} \\ \mathcal{A}_{b, b} x_{b}^{2} & \mathcal{A}_{b, b} x_{b} y_{b} & \mathcal{A}_{b, b} x_{b} y_{b} & \mathcal{A}_{b, b} y_{b}^{2}\end{array}\right) \rho_{\mathrm{sc}}(\boldsymbol{x}) \boldsymbol{S}\left(\theta_{\mathrm{sc}}\right) \mathrm{d} \boldsymbol{x}$,

where we define

$$
\mathcal{A}_{i, j}=\left(1 / 16 \pi^{2} R_{\boldsymbol{x}, s}^{2}\right)\left(F^{\mathrm{sc}}\left(k \widehat{\boldsymbol{R}}_{\boldsymbol{x}, s} \cdot \widehat{\boldsymbol{e}}_{\mathrm{sc}}\right)\right)^{2} F^{i}\left(k \widehat{\boldsymbol{R}}_{\boldsymbol{x}, s} \cdot \widehat{\boldsymbol{e}}_{i}\right) F^{j}\left(k \widehat{\boldsymbol{R}}_{\boldsymbol{x}, s} \cdot \widehat{\boldsymbol{e}}_{j}\right) P_{i}(k)
$$

for $i=a, b$ and $j=a, b$.

The SAR imaging problem is to determine the unknowns $\rho(\boldsymbol{x})$ and $\boldsymbol{S}\left(\theta_{\mathrm{sc}}(\boldsymbol{x})\right)$ from measurements of $\boldsymbol{D}$.

2.2.5. Simplifications. We ultimately aim to have a forward model that is of the form $\boldsymbol{D}=\mathcal{F}^{T}[\boldsymbol{T}]+\mathcal{F}^{C}[\boldsymbol{C}]+\boldsymbol{n}$, where $\boldsymbol{T}$ is a function describing the target, $\boldsymbol{C}$ describes clutter, the operators $\mathcal{F}^{T}$ and $\mathcal{F}^{C}$ are linear operators and $\boldsymbol{n}$ is noise.

In the case when $\mathcal{F}^{T}$ and $\mathcal{F}^{C}$ are Fourier integral operators (which are linear), we can write down an analytic expression for an approximate inverse operator [7].

The model (18) is linear in $\rho$, but nonlinear in $\theta_{\mathrm{sc}}$ because the amplitudes $\mathcal{A}_{i, j}$ of (19) contain $\theta_{\mathrm{sc}}$. In order to remove this nonlinearity, we will make assumptions about the radiation patterns of the target and clutter [41].

\subsection{Scattering model for the target}

For an extended target, we assume that we obtain a strong return from the scatterer only when the orientation of the target is perpendicular to the look direction:

$$
F^{T}\left(k \widehat{\boldsymbol{R}}_{\boldsymbol{x}, s}^{i} \cdot \widehat{\boldsymbol{e}}_{T}\right)= \begin{cases}1 & \text { if } \widehat{\boldsymbol{R}}_{\boldsymbol{x}, \mathrm{s}} \cdot \widehat{\boldsymbol{e}}_{\mathrm{T}} \approx 0 \\ 0 & \text { otherwise }\end{cases}
$$

We change the subscript and superscript to the letter $T$ to indicate we are considering a target scatterer.

Using this directional assumption, we can simplify expression (12):

$$
\begin{aligned}
{\left[\widehat{\boldsymbol{R}}_{\boldsymbol{x}, s} \times\left(\widehat{\boldsymbol{R}}_{\boldsymbol{x}, s} \times \widehat{\boldsymbol{e}}_{a}\right)\right] \cdot \widehat{\boldsymbol{e}}_{T}\left[\widehat{\boldsymbol{R}}_{\boldsymbol{x}, s} \times\left(\widehat{\boldsymbol{R}}_{\boldsymbol{x}, s} \times \widehat{\boldsymbol{e}}_{b}\right)\right] \cdot \widehat{\boldsymbol{e}}_{T} } & =\left(-\widehat{\boldsymbol{e}}_{T} \cdot \widehat{\boldsymbol{e}}_{a}\right)\left(-\widehat{\boldsymbol{e}}_{T} \cdot \widehat{\boldsymbol{e}}_{b}\right) \\
& =\left[\widehat{\boldsymbol{e}}_{a} \otimes \widehat{\boldsymbol{e}}_{b}\right]:\left[\widehat{\boldsymbol{e}}_{T} \otimes \widehat{\boldsymbol{e}}_{T}\right]
\end{aligned}
$$


Therefore, we write

$$
\left(\begin{array}{c}
\left(\boldsymbol{R}_{a}^{\perp} \otimes \boldsymbol{R}_{a}^{\perp}\right):\left(\widehat{\boldsymbol{e}}_{T} \otimes \widehat{\boldsymbol{e}}_{T}\right) \\
\left(\boldsymbol{R}_{a}^{\perp} \otimes \boldsymbol{R}_{b}^{\perp}\right):\left(\widehat{\boldsymbol{e}}_{T} \otimes \widehat{\boldsymbol{e}}_{T}\right) \\
\left(\boldsymbol{R}_{b}^{\perp} \otimes \boldsymbol{R}_{a}^{\perp}\right):\left(\widehat{\boldsymbol{e}}_{T} \otimes \widehat{\boldsymbol{e}}_{T}\right) \\
\left(\boldsymbol{R}_{b}^{\perp} \otimes \boldsymbol{R}_{b}^{\perp}\right):\left(\widehat{\boldsymbol{e}}_{T} \otimes \widehat{\boldsymbol{e}}_{T}\right)
\end{array}\right)=\left(\begin{array}{cccc}
a_{1}^{2} & a_{1} a_{2} & a_{1} a_{2} & a_{2}^{2} \\
a_{1} b_{1} & a_{1} b_{2} & a_{2} b_{1} & a_{2} b_{2} \\
a_{1} b_{1} & a_{2} b_{1} & a_{1} b_{2} & a_{2} b_{2} \\
b_{1}^{2} & b_{1} b_{2} & b_{1} b_{2} & b_{2}^{2}
\end{array}\right)\left(\begin{array}{c}
\cos ^{2} \theta_{T} \\
\cos \theta_{T} \sin \theta_{T} \\
\sin \theta_{T} \cos \theta_{T} \\
\sin ^{2} \theta_{T}
\end{array}\right),
$$

where we let $\widehat{\boldsymbol{e}}_{a}=\left(a_{1}, a_{2}, 0\right)$ and $\widehat{\boldsymbol{e}}_{b}=\left(b_{1}, b_{2}, 0\right)$. Note we have used the triple product, or BAC-CAB, identity as in (12) and the tensor notation as in (13).

The assumption (20) converts (18) to the form

$$
\boldsymbol{D}^{T}(k, s)=\mathcal{F}^{T}[\boldsymbol{T}(\boldsymbol{x})]=\int \mathrm{e}^{2 \mathrm{i} k R_{x, s}} \underline{A}^{T}(k, s, \boldsymbol{x}) \boldsymbol{T}(\boldsymbol{x}) \mathrm{d} \boldsymbol{x},
$$

where we define $\boldsymbol{T}(\boldsymbol{x})=\rho_{T}(\boldsymbol{x}) \boldsymbol{S}\left(\theta_{T}\right)$ as the target vector field. The amplitude $\underline{A}^{T}$ now depends only on $\widehat{\boldsymbol{e}}_{a}$ and $\widehat{\boldsymbol{e}}_{b}$ and not $\widehat{\boldsymbol{R}}_{\boldsymbol{x}, s}$ :

$$
\underline{A}^{T}(k, s, \boldsymbol{x})=\left(\begin{array}{cccc}
\tilde{\mathcal{A}}_{a, a} a_{1}^{2} & \tilde{\mathcal{A}}_{a, a} a_{1} a_{2} & \tilde{\mathcal{A}}_{a, a} a_{1} a_{2} & \tilde{\mathcal{A}}_{a, a} a_{2}^{2} \\
\tilde{\mathcal{A}}_{a, b} a_{1} b_{1} & \tilde{\mathcal{A}}_{a, b} a_{1} b_{2} & \tilde{\mathcal{A}}_{a, b} a_{2} b_{1} & \tilde{\mathcal{A}}_{a, b} a_{2} b_{2} \\
\tilde{\mathcal{A}}_{b, a} a_{1} b_{1} & \tilde{\mathcal{A}}_{b, a} a_{2} b_{1} & \tilde{\mathcal{A}}_{b, a} a_{1} b_{2} & \tilde{\mathcal{A}}_{b, a} a_{2} b_{2} \\
\tilde{\mathcal{A}}_{b, b} b_{1}^{2} & \tilde{\mathcal{A}}_{b, b} b_{1} b_{2} & \tilde{\mathcal{A}}_{b, b} b_{1} b_{2} & \tilde{\mathcal{A}}_{b, b} b_{2}^{2}
\end{array}\right),
$$

where

$$
\tilde{\mathcal{A}}_{i, j}=\left(1 / 16 \pi^{2} R_{\boldsymbol{x}, s}^{2}\right) F^{i}\left(k \widehat{\boldsymbol{R}}_{\boldsymbol{x}, s} \cdot \widehat{\boldsymbol{e}}_{i}\right) F^{j}\left(k \widehat{\boldsymbol{R}}_{\boldsymbol{x}, s} \cdot \widehat{\boldsymbol{e}}_{j}\right) P_{i}(k)
$$

for $i=a, b$ and $j=a, b$. We clearly see that the amplitude matrix no longer depends on the unknown quantity $\widehat{\boldsymbol{e}}_{T}$, and therefore we obtain a linear forward model for the target data.

\subsection{Scattering model for clutter}

For clutter scatterers, we assume that the scattering is isotropic:

$$
F^{C}\left(k \widehat{\boldsymbol{R}}_{\boldsymbol{x}, s} \cdot \widehat{\boldsymbol{e}}_{C}\right)=1
$$

Again, this removes the nonlinearity from the forward model. We obtain the following forward model for clutter data:

$$
\boldsymbol{D}^{C}(k, s)=\mathcal{F}^{C}[\boldsymbol{C}](k, s)=\int \mathrm{e}^{2 \mathrm{i} k R_{x, s}} \underline{A}^{C}(k, s, \boldsymbol{x}) \boldsymbol{C}(\boldsymbol{x}) \mathrm{d} \boldsymbol{x},
$$

where we let the vector field that describes the clutter be $\boldsymbol{C}(\boldsymbol{x})=\rho_{C}(\boldsymbol{x}) \boldsymbol{S}_{C}\left(\theta_{C}\right)$. Note $\rho_{C}(\boldsymbol{x})$ is the clutter scattering strength at $\boldsymbol{x}$, and $\boldsymbol{S}_{C}\left(\theta_{C}\right)$ is the clutter scattering vector which depends on the orientation of the clutter dipole element at location $\boldsymbol{x}$. The amplitude matrix has the form

$$
\underline{A}^{C}(k, s, \boldsymbol{x})=\left(\begin{array}{cccc}
\tilde{\mathcal{A}}_{a, a} x_{a}^{2} & \tilde{\mathcal{A}}_{a, a} x_{a} y_{a} & \tilde{\mathcal{A}}_{a, a} x_{a} y_{a} & \tilde{\mathcal{A}}_{a, a} y_{a}^{2} \\
\tilde{\mathcal{A}}_{a, b} x_{a} x_{b} & \tilde{\mathcal{A}}_{a, b} x_{a} y_{b} & \tilde{\mathcal{A}}_{a, b} y_{a} x_{b} & \tilde{\mathcal{A}}_{a, b} y_{a} y_{b} \\
\tilde{\mathcal{A}}_{b, a} x_{a} x_{b} & \tilde{\mathcal{A}}_{b, a} y_{a} x_{b} & \tilde{\mathcal{A}}_{b, a} x_{a} y_{b} & \tilde{\mathcal{A}}_{b, a} y_{b} y_{b} \\
\tilde{\mathcal{A}}_{b, b} x_{b}^{2} & \tilde{\mathcal{A}}_{b, b} x_{b} y_{b} & \tilde{\mathcal{A}}_{b, b} x_{b} y_{b} & \tilde{\mathcal{A}}_{b, b} y_{b}^{2}
\end{array}\right)
$$

where $\tilde{\mathcal{A}}_{i, j}$ for $i=a, b$ and $j=a, b$ are defined as in equation (25). Note that while we define the clutter vector field as $\boldsymbol{C}(\boldsymbol{x})=\rho_{C}(\boldsymbol{x}) \boldsymbol{S}_{C}\left(\theta_{C}\right)$, the associated scattering vector (as in equation (15)) includes the cross products of (17). Therefore, the zero-endfire character of dipoles is included for clutter dipoles.

The two forward operators $\mathcal{F}^{C}$ and $\mathcal{F}^{T}$ are different, but under the assumptions above, both are now linear operators. 


\subsection{Statistical forward model}

We combine the target and clutter data with measurement noise $\boldsymbol{n}$ to obtain the full forward model expression. That is, we expect our collected data $\boldsymbol{D}$ to be of the form

$\boldsymbol{D}(k, s)=\mathcal{F}^{T}[\boldsymbol{T}](k, s)+\mathcal{F}^{C}[\boldsymbol{C}](k, s)+\boldsymbol{n}(k, s)=\boldsymbol{D}^{T}(k, s)+\boldsymbol{D}^{C}(k, s)+\boldsymbol{n}(k, s)$.

More specifically, we have

$\boldsymbol{D}(k, s)=\int \mathrm{e}^{2 \mathrm{i} k R_{\boldsymbol{x}, s}} \underline{A}^{T}(k, s, \boldsymbol{x}) \boldsymbol{T}(\boldsymbol{x}) \mathrm{d} \boldsymbol{x}+\int \mathrm{e}^{2 \mathrm{i} k R_{\boldsymbol{x}, s}} \underline{A}^{C}(k, s, \boldsymbol{x}) \boldsymbol{C}(\boldsymbol{x}) \mathrm{d} \boldsymbol{x}+\boldsymbol{n}(k, s)$,

where we assume $\boldsymbol{n}$ is a $4 \times 1$ vector.

We also make the assumption that the target vector field $\boldsymbol{T}(\boldsymbol{x})$, the clutter vector field $\boldsymbol{C}(\boldsymbol{x})$ and the noise vector field $\boldsymbol{n}(k, s)$ are all second-order stochastic processes, in the sense that each element of the covariance matrix is finite. We have already specified a somewhat rigid form for the target vector field, namely $\boldsymbol{T}(\boldsymbol{x})=\rho_{T}(\boldsymbol{x}) \boldsymbol{S}\left(\theta_{T}\right)$. The functional form of $\boldsymbol{S}$ is assumed to be known and the parameter $\theta_{T}$ is stochastic. We leave the form of the scattering strength unspecified, and therefore we will eventually need to define a probability distribution describing the stochastic nature of $\rho_{T}(\boldsymbol{x})$. For now we do not assign specific distributions to these random quantities. In our numerical experiments, we will specify distributions and they will be discussed in detail in section 4 .

We make the following statistical assumptions on $\boldsymbol{T}, \boldsymbol{C}$ and $\boldsymbol{n}$. For the first-order statistics, we assume

$$
\begin{aligned}
& E[\boldsymbol{T}(\boldsymbol{x})]=\boldsymbol{\mu}(\boldsymbol{x}) \\
& E[\boldsymbol{C}(\boldsymbol{x})]=\mathbf{0} \\
& E[\boldsymbol{n}(k, s)]=\mathbf{0},
\end{aligned}
$$

where $\boldsymbol{\mu}(\boldsymbol{x})=\left[E\left[T_{a, a}(\boldsymbol{x})\right], E\left[T_{a, b}(\boldsymbol{x})\right], E\left[T_{b, a}(\boldsymbol{x})\right], E\left[T_{b, b}(\boldsymbol{x})\right]\right]$ and where $\mathbf{0}$ is the $4 \times 1$ zero vector. We also specify the autocovariance matrices for $\boldsymbol{T}, \boldsymbol{C}$ and $\boldsymbol{n}$, where we define the $(l, m)$ th entry as follows

$$
\begin{aligned}
& C_{l, m}^{T}\left(\boldsymbol{x}, \boldsymbol{x}^{\prime}\right)=E\left[\left(T_{l}(\boldsymbol{x})-\mu_{l}(\boldsymbol{x}) \overline{\left(T_{m}\left(\boldsymbol{x}^{\prime}\right)-\mu_{m}(\boldsymbol{x})\right)}\right]\right. \\
& \mathcal{R}_{l, m}^{C}\left(\boldsymbol{x}, \boldsymbol{x}^{\prime}\right)=E\left[C_{l}(\boldsymbol{x}) \overline{C_{m}\left(\boldsymbol{x}^{\prime}\right)}\right] \\
& \mathcal{S}_{l, m}^{n}\left(k, s ; k^{\prime}, s^{\prime}\right)=E\left[n_{l}(k, s) \overline{n_{m}\left(k^{\prime}, s^{\prime}\right)}\right]
\end{aligned}
$$

where here $l=a a, a b, b a, b b$ and $m=a a, a b, b a, b b$. We assume the Fourier transforms for each element of $\underline{C}^{T}$ and $\underline{\mathcal{R}}^{C}$ exist, and we assume that the target, clutter and noise are all mutually statistically independent.

\section{Image formation in the presence of noise and clutter}

In order to form an image of the target, we will use a filtered-backprojection-based reconstruction method. Specifically, we apply the backprojection operator $\mathcal{K}$ to the measured data to form an image $\boldsymbol{I}$ of the target, i.e.

$$
\boldsymbol{I}(\boldsymbol{z})=(\mathcal{K} \boldsymbol{D})(\boldsymbol{z})=\int \mathrm{e}^{-\mathrm{i} 2 k \boldsymbol{R}_{z, s}} \underline{Q}(z, s, k) \boldsymbol{D}(k, s) \mathrm{d} k \mathrm{~d} s,
$$


where $\boldsymbol{I}(\boldsymbol{z})=\left[I_{a, a}(\boldsymbol{z}), I_{a, b}(\boldsymbol{z}), I_{b, a}(\boldsymbol{z}), I_{b, b}(\boldsymbol{z})\right]$. We define $\underline{Q}$ as a $4 \times 4$ filter matrix. The filter $\underline{Q}$ can be chosen in a variety of ways. One method attempts to provide a point-spread function (or ambiguity function) that most closely resembles a delta function [7, 27]. This method works well in the case when we assume that the target vector field can be described deterministically. We will instead consider a statistical criterion for selecting the optimal filter $Q$. In particular, we will attempt to minimize the MSE between the reconstructed image $\boldsymbol{I}$ and the actual target vector field $\boldsymbol{T}$. This method seeks to minimize the effect of noise and clutter on the resulting image while preserving the strength of the singularities of the target vector field. This method was first described for the case of standard SAR in [42].

We begin by first defining an error process

$$
\boldsymbol{E}(\boldsymbol{z})=\boldsymbol{I}(\boldsymbol{z})-\mathcal{I}_{\Omega} \boldsymbol{T}(\boldsymbol{z}),
$$

where $\mathcal{I}_{\Omega} \boldsymbol{T}(\boldsymbol{z})$ is the ideal $L^{2}$ image that can be obtained from our SAR system, namely

$$
\mathcal{I}_{\Omega} \boldsymbol{T}(\boldsymbol{z})=\int \underline{\tilde{\chi}}_{\Omega}(z, \xi) \mathrm{e}^{\mathrm{i}\left(z^{\prime}-z\right) \cdot \xi} \boldsymbol{T}\left(z^{\prime}\right) \mathrm{d} \xi \mathrm{d} z^{\prime},
$$

where $\tilde{\chi}_{\Omega}(z, \xi)$ is a smoothed version of the characteristic function of $\Omega$. Here $\Omega$ is the set of target Fourier coefficients that can be obtained from our measurements; this is discussed in more detail below. The smoothing is done to avoid ringing.

We also define the MSE as

$$
\mathcal{J}(\underline{Q})=\int E\left[|\boldsymbol{E}(\boldsymbol{z})|^{2}\right] \mathrm{d} z=\int E\left[\left(\boldsymbol{E}(\boldsymbol{z})^{\dagger}(\boldsymbol{E}(\boldsymbol{z}))\right] \mathrm{d} z,\right.
$$

where $\boldsymbol{E}^{\dagger}$ denotes the complex conjugate transpose of the vector $\boldsymbol{E}$. Note that we have

$$
\mathcal{J}(\underline{Q})=\mathcal{V}(\underline{Q})+\mathcal{B}(\underline{Q})
$$

where

$$
\begin{aligned}
& \mathcal{V}(\underline{Q})=\int E\left[|\boldsymbol{E}(\boldsymbol{z})-E[\boldsymbol{E}(\boldsymbol{z})]|^{2}\right] \mathrm{d} z \\
& \mathcal{B}(\underline{Q})=\int\left|E[\boldsymbol{I}(\boldsymbol{z})]-E\left[\mathcal{I}_{\Omega} \boldsymbol{T}(\boldsymbol{z})\right]\right|^{2} \mathrm{~d} \boldsymbol{z} .
\end{aligned}
$$

Here $\mathcal{V}$ is the total variance of $\boldsymbol{E}(\boldsymbol{z})$ and $\mathcal{B}$ is the $L^{2}$ norm of the bias. It is well known that the MSE is the sum of variance and bias and that when we attempt to minimize the MSE there is always a tradeoff between minimizing variance and bias. Minimizing the MSE will come at a cost with respect to visible singularities of the target vector field. These singularities, which for example include target edges, are critical in identifying a target in an image. As discussed in [42], the strengths of these singularities may be partially suppressed in our attempts to suppress the clutter and noise contributions to the image. The location and orientation of the visible singularities, however, will be maintained, because, as we will see, the image-fidelity operator $\mathcal{K F}^{T}$ is a pseudodifferential operator.

Lemma. Let $\boldsymbol{D}$ be given by (29), where the amplitudes $\underline{A}^{T}$ and $\underline{A}^{C}$ satisfy symbol estimates [15] and let I be given by (37). Assume $\underline{S}^{n}$ is given by (36) and define $\underline{S}_{T}, \underline{S}_{C}$ and $\underline{M}$ as follows:

$$
\begin{aligned}
& \underline{C}^{T}\left(\boldsymbol{x}, \boldsymbol{x}^{\prime}\right)=\int \mathrm{e}^{-\mathrm{i} x \cdot \zeta} \mathrm{e}^{\mathrm{i} x^{\prime} \cdot \zeta^{\prime}} \underline{S}_{T}\left(\boldsymbol{\zeta}, \boldsymbol{\zeta}^{\prime}\right) \mathrm{d} \zeta \mathrm{d} \boldsymbol{\zeta}^{\prime} \\
& \underline{\mathcal{R}}^{C}\left(\boldsymbol{x}, \boldsymbol{x}^{\prime}\right)=\int \mathrm{e}^{-\mathrm{i} x \cdot \zeta} \mathrm{e}^{\mathrm{i} x^{\prime} \cdot \zeta^{\prime}} \underline{S}_{C}\left(\boldsymbol{\zeta}, \boldsymbol{\zeta}^{\prime}\right) \mathrm{d} \boldsymbol{\zeta} \mathrm{d} \boldsymbol{\zeta}^{\prime} \\
& \boldsymbol{\mu}(\boldsymbol{x}) \boldsymbol{\mu}^{\dagger}\left(\boldsymbol{x}^{\prime}\right)=\int \mathrm{e}^{-\mathrm{i} x \cdot \zeta} \mathrm{e}^{\mathrm{i} x^{\prime} \cdot \boldsymbol{\zeta}^{\prime}} \underline{M}\left(\boldsymbol{\zeta}, \boldsymbol{\zeta}^{\prime}\right) \mathrm{d} \zeta \mathrm{d} \boldsymbol{\zeta}^{\prime},
\end{aligned}
$$


where the integrations are defined element-wise. Then

$$
\mathcal{J}(\underline{Q})=\tilde{\mathcal{J}}_{T}(\underline{Q})+\mathcal{B}(\underline{Q})+\mathcal{J}_{C}(\underline{Q})+\mathcal{J}_{n}(\underline{Q})
$$

and the expected values of the leading-order singularities of each term in (47) are

$$
\begin{aligned}
& \tilde{\mathcal{J}}_{T}(\underline{Q}) \approx \int \mathrm{e}^{\mathrm{i} \boldsymbol{x} \cdot\left(\boldsymbol{\zeta}^{\prime}-\zeta\right)} \operatorname{tr}\left[\left\{\underline{Q}\left(\boldsymbol{x}, \boldsymbol{\zeta}^{\prime}\right) \underline{A}^{T}\left(\boldsymbol{x}, \boldsymbol{\zeta}^{\prime}\right) \eta\left(\boldsymbol{x}, \boldsymbol{x}, \boldsymbol{\zeta}^{\prime}\right)-\underline{\tilde{\chi}}_{\Omega}\left(\boldsymbol{x}, \boldsymbol{\zeta}^{\prime}\right)\right\}^{\dagger}\right. \\
& \left.\times\left\{\underline{Q}\left(\boldsymbol{x}, \boldsymbol{\zeta}^{\prime}\right) \underline{A}^{T}\left(\boldsymbol{x}, \boldsymbol{\zeta}^{\prime}\right) \eta\left(\boldsymbol{x}, \boldsymbol{x}, \boldsymbol{\zeta}^{\prime}\right)-\underline{\tilde{x}}_{\Omega}\left(\boldsymbol{x}, \boldsymbol{\zeta}^{\prime}\right)\right\} \underline{S}_{T}\left(\boldsymbol{\zeta}, \boldsymbol{\zeta}^{\prime}\right)\right] \mathrm{d} \boldsymbol{x} \mathrm{d} \boldsymbol{\zeta} \mathrm{d} \boldsymbol{\zeta}^{\prime}, \\
& \mathcal{J}_{C}(\underline{Q}) \approx \int \mathrm{e}^{\mathrm{i} \boldsymbol{x} \cdot\left(\boldsymbol{\zeta}^{\prime}-\zeta\right)} \operatorname{tr}\left[\left\{\underline{Q}\left(\boldsymbol{x}, \boldsymbol{\zeta}^{\prime}\right) \underline{A}^{C}\left(\boldsymbol{x}, \boldsymbol{\zeta}^{\prime}\right) \eta\left(\boldsymbol{x}, \boldsymbol{x}, \boldsymbol{\zeta}^{\prime}\right)\right\}^{\dagger}\right. \\
& \left.\times\left\{\underline{Q}\left(\boldsymbol{x}, \boldsymbol{\zeta}^{\prime}\right) \underline{A}^{C}\left(\boldsymbol{x}, \boldsymbol{\zeta}^{\prime}\right) \eta\left(\boldsymbol{x}, \boldsymbol{x}, \boldsymbol{\zeta}^{\prime}\right)\right\} \underline{S}_{C}\left(\boldsymbol{\zeta}, \boldsymbol{\zeta}^{\prime}\right)\right] \mathrm{d} \boldsymbol{x} \mathrm{d} \boldsymbol{\zeta} \mathrm{d} \boldsymbol{\zeta}^{\prime}, \\
& \mathcal{B}(\underline{Q}) \approx \int \mathrm{e}^{\mathrm{i} \boldsymbol{x} \cdot\left(\boldsymbol{\zeta}^{\prime}-\zeta\right)} \operatorname{tr}\left[\left\{\underline{Q}\left(\boldsymbol{x}, \boldsymbol{\zeta}^{\prime}\right) \underline{A}^{T}\left(\boldsymbol{x}, \boldsymbol{\zeta}^{\prime}\right) \eta\left(\boldsymbol{x}, \boldsymbol{x}, \boldsymbol{\zeta}^{\prime}\right)-\underline{\tilde{\chi}}_{\Omega}\left(\boldsymbol{x}, \boldsymbol{\zeta}^{\prime}\right)\right\}^{\dagger}\right. \\
& \left.\times\left\{\underline{Q}\left(\boldsymbol{x}, \boldsymbol{\zeta}^{\prime}\right) \underline{A}^{T}\left(\boldsymbol{x}, \boldsymbol{\zeta}^{\prime}\right) \eta\left(\boldsymbol{x}, \boldsymbol{x}, \boldsymbol{\zeta}^{\prime}\right)-\underline{\tilde{\chi}}_{\Omega}\left(\boldsymbol{x}, \boldsymbol{\zeta}^{\prime}\right)\right\} \underline{M}\left(\boldsymbol{\zeta}, \boldsymbol{\zeta}^{\prime}\right)\right] \mathrm{d} \boldsymbol{x} \mathrm{d} \boldsymbol{\zeta} \mathrm{d} \boldsymbol{\zeta}^{\prime}, \\
& \mathcal{J}_{n}(\underline{Q})=\int \operatorname{tr}\left[\underline{Q}^{\dagger}(z, \xi) \underline{Q}(z, \xi) \underline{\tilde{S}}^{n}(\xi)\right] \eta(z, z, \xi) \mathrm{d} \boldsymbol{\xi} \mathrm{d} z .
\end{aligned}
$$

Here 'leading order' is in the microlocal sense [15, 33], meaning that the higher order terms are smoother than the leading-order one, and $\approx$ indicates that we are taking the expected $L^{2}$ norm of the leading-order term.

Proof. The MSE $\mathcal{J}(\underline{Q})$ is given by the expression

$$
\mathcal{J}(\underline{Q})=\int E\left[\left|\left(\mathcal{K}\left(\mathcal{F}^{T}(\boldsymbol{T})+\mathcal{F}^{C}(\boldsymbol{C})+\boldsymbol{n}\right)\right)(\boldsymbol{z})-\mathcal{I}_{\Omega} \boldsymbol{T}(\boldsymbol{z})\right|^{2}\right] \mathrm{d} \boldsymbol{z} .
$$

Because we have assumed that $\boldsymbol{T}, \boldsymbol{C}$ and $\boldsymbol{n}$ are mutually statistically independent, this MSE can be written as a sum of three terms:

$$
\mathcal{J}(\underline{Q})=\mathcal{J}_{T}(\underline{Q})+\mathcal{J}_{C}(\underline{Q})+\mathcal{J}_{n}(\underline{Q})
$$

where

$$
\begin{aligned}
& \mathcal{J}_{T}(\underline{Q})=\int E\left[\left|\left(\mathcal{K}\left(\mathcal{F}^{T}\right)-\mathcal{I}_{\Omega}\right)(\boldsymbol{T})(\boldsymbol{z})\right|^{2}\right] \mathrm{d} z, \\
& \mathcal{J}_{C}(\underline{Q})=\int E\left[\left|\mathcal{K}\left(\mathcal{F}^{C}(\boldsymbol{C})\right)(\boldsymbol{z})\right|^{2}\right] \mathrm{d} z, \\
& \mathcal{J}_{n}(\underline{Q})=\int E\left[|\mathcal{K}(\boldsymbol{n})(\boldsymbol{z})|^{2}\right] \mathrm{d} z .
\end{aligned}
$$

First we simplify the expression for $\mathcal{J}_{T}(\underline{Q})$. The first term of (54) is dependent on the image-fidelity operator $\mathcal{K} \mathcal{F}^{T}$ applied to $\boldsymbol{T}$ :

$$
\mathcal{K}\left(\mathcal{F}^{T} \boldsymbol{T}\right)(\boldsymbol{z})=\int \mathrm{e}^{-\mathrm{i} 2 k\left(\boldsymbol{R}_{z, s}-\boldsymbol{R}_{x, s}\right)} \underline{Q}(z, s, k) \underline{A}^{T}(\boldsymbol{x}, s, k) \boldsymbol{T}(\boldsymbol{x}) \mathrm{d} \boldsymbol{x} \mathrm{d} k \mathrm{~d} s .
$$

From the method of stationary phase in the variables $(s, k)$, we know that the main contributions to the integral (57) come from the critical points of the phase. We have assumed that the antenna beam pattern is such that only the critical points for which $\boldsymbol{x}=\boldsymbol{z}$ contribute to (57), because others are not illuminated by the antenna. 
In order to obtain a phase that resembles that of a delta function, namely $(\boldsymbol{x}-\boldsymbol{z}) \cdot \boldsymbol{\xi}$, we expand the phase about the point $\boldsymbol{x}=\boldsymbol{z}$. This phase ensures our image-fidelity operator is a pseudodifferential operator. We use the mean value theorem as in [7, 27]:

$$
\begin{aligned}
f(\boldsymbol{x})-f(\boldsymbol{z}) & =\int_{0}^{1} \frac{\mathrm{d}}{\mathrm{d} \mu} f(\boldsymbol{z}+\mu(\boldsymbol{x}-\boldsymbol{z})) \mathrm{d} \mu \\
& =\left.(\boldsymbol{x}-\boldsymbol{z}) \cdot \int_{0}^{1} \nabla f\right|_{z+\mu(\boldsymbol{x}-z)} \mathrm{d} \mu=(\boldsymbol{x}-\boldsymbol{z}) \cdot \boldsymbol{\Xi}(\boldsymbol{x}, \boldsymbol{z}, s, k),
\end{aligned}
$$

where in our case $f(z)=2 k \boldsymbol{R}_{z, s}$. We now perform the Stolt change of variables from $(s, k)$ to $\boldsymbol{\xi}=\boldsymbol{\Xi}(\boldsymbol{x}, \boldsymbol{z}, s, k)$, where $\Xi$ is defined in the last line of (58). Therefore, we have

$\mathcal{K}\left(\mathcal{F}^{T} T\right)(\boldsymbol{z}) \sim \int \mathrm{e}^{\mathrm{i}(\boldsymbol{x}-\boldsymbol{z}) \cdot \boldsymbol{\xi}} \underline{Q}(\boldsymbol{z}, s(\boldsymbol{\xi}), k(\boldsymbol{\xi})) \underline{A}^{T}(\boldsymbol{x}, s(\boldsymbol{\xi}), k(\boldsymbol{\xi})) \boldsymbol{T}(\boldsymbol{x}) \eta(\boldsymbol{x}, \boldsymbol{z}, \boldsymbol{\xi}) \mathrm{d} \boldsymbol{x} \mathrm{d} \boldsymbol{\xi}$,

where $\eta$ is the Jacobian resulting from the change of variables, sometimes called the Beylkin determinant [2]. In (59), we give only the leading-order term; the higher order terms are smoother.

We can now substitute (59) into our expression for $\mathcal{J}_{T}(\underline{Q}),(67)$. We have

$\mathcal{J}_{T}(\underline{Q}) \approx \int E\left|\int \mathrm{e}^{\mathrm{i}(\boldsymbol{x}-z) \cdot \xi}\left\{\underline{Q}(\boldsymbol{z}, \boldsymbol{\xi}) \underline{A}^{T}(\boldsymbol{x}, \boldsymbol{\xi}) \eta(\boldsymbol{x}, \boldsymbol{z}, \boldsymbol{\xi})-\underline{\tilde{\chi}}_{\Omega}(\boldsymbol{z}, \boldsymbol{\xi})\right\} \boldsymbol{T}(\boldsymbol{x}) \mathrm{d} \boldsymbol{\xi} \mathrm{d} \boldsymbol{x}\right|^{2} \mathrm{~d} \boldsymbol{z}$,

where $\approx$ indicates that we are taking the expected $L^{2}$ norm of the leading-order term, namely that obtained from (59).

We note that (60) involves a sum of scalar terms of the form

$$
\Delta=\left\langle\mathcal{A}_{1} T, \mathcal{A}_{2} T\right\rangle_{L^{2}}=\left\langle\mathcal{A}_{2}^{\dagger} \mathcal{A}_{1} T, T\right\rangle_{L^{2}},
$$

where $\mathcal{A}_{1}$ is the pseudodifferential operator

$$
\left(\mathcal{A}_{1} T\right)(z)=\int \mathrm{e}^{\mathrm{i}(\boldsymbol{x}-z) \cdot \xi} \tilde{A}_{1}(\boldsymbol{z}, \boldsymbol{x}, \boldsymbol{\xi}) \mathrm{d} \boldsymbol{\xi} T(\boldsymbol{x}) \mathrm{d} \boldsymbol{x}
$$

and $\mathcal{A}_{2}$ is defined similarly. A standard result in the theory of pseudodifferential operators [15, $33]$ tells us that an operator of the form (62) can be written as

$\mathcal{A}_{1} T(\boldsymbol{z})=\int \mathrm{e}^{\mathrm{i}(\boldsymbol{x}-z) \cdot \xi} \tilde{A}_{1}(\boldsymbol{z}, \boldsymbol{x}, \boldsymbol{\xi}) \mathrm{d} \boldsymbol{\xi} T(\boldsymbol{x}) \mathrm{d} \boldsymbol{x}=\int \mathrm{e}^{\mathrm{i}(\boldsymbol{x}-z) \cdot \xi} p(z, \xi) \mathrm{d} \boldsymbol{\xi} T(\boldsymbol{x}) \mathrm{d} \boldsymbol{x}$

where $p(z, \xi)=\mathrm{e}^{-\mathrm{i} z \cdot \xi} \mathcal{A}_{1}\left(\mathrm{e}^{\mathrm{i} z \cdot \xi}\right)$. The symbol $p$ has an asymptotic expansion

$$
\left.p(z, \xi) \sim \sum_{\alpha \geqslant 0} \frac{\mathrm{i}^{|\alpha|}}{\alpha !} D_{\xi}^{\alpha} D_{x}^{\alpha} \tilde{A}_{1}(z, \boldsymbol{x}, \boldsymbol{\xi})\right|_{z=x}
$$

where $\alpha$ is a multi-index. In other words, the leading-order term of $p(z, \xi)$ is simply $\tilde{A}_{1}(\boldsymbol{z}, \boldsymbol{z}, \boldsymbol{\xi})$. Moreover, according to the symbol calculus [15,33], the leading-order term of $\mathcal{A}_{2}^{\dagger} \mathcal{A}_{1}$ can be written as

$$
\left(\mathcal{A}_{2}^{\dagger} \mathcal{A}_{1} T\right)(z) \approx \int \mathrm{e}^{\mathrm{i}(\boldsymbol{x}-z) \cdot \xi} \tilde{\tilde{A}}_{2}(\boldsymbol{z}, \boldsymbol{z}, \boldsymbol{\xi}) \tilde{A}_{1}(z, z, \xi) \mathrm{d} \boldsymbol{\xi} T(\boldsymbol{x}) \mathrm{d} \boldsymbol{x} .
$$

This implies that the leading-order contribution to (60) is

$$
\begin{aligned}
\mathcal{J}_{T}\left(\underline{Q)} \approx \int E\right. & {\left[\int \mathrm{e}^{\mathrm{i}\left(\boldsymbol{x}-\boldsymbol{x}^{\prime}\right) \cdot \boldsymbol{\xi}} \boldsymbol{T}^{\dagger}(\boldsymbol{x})\left\{\underline{Q}\left(\boldsymbol{x}^{\prime}, \boldsymbol{\xi}\right) \underline{A}^{T}\left(\boldsymbol{x}^{\prime}, \boldsymbol{\xi}\right) \eta\left(\boldsymbol{x}^{\prime}, \boldsymbol{x}^{\prime}, \boldsymbol{\xi}\right)-\underline{\tilde{\chi}}_{\Omega}\left(\boldsymbol{x}^{\prime}, \boldsymbol{\xi}\right)\right\}^{\dagger}\right.} \\
\times & \left.\left\{\underline{Q}\left(\boldsymbol{x}^{\prime}, \boldsymbol{\xi}\right) \underline{A}^{T}\left(\boldsymbol{x}^{\prime}, \boldsymbol{\xi}\right) \eta\left(\boldsymbol{x}^{\prime}, \boldsymbol{x}^{\prime}, \boldsymbol{\xi}\right)-\underline{\tilde{\chi}}_{\Omega}\left(\boldsymbol{x}^{\prime}, \boldsymbol{\xi}\right)\right\} \boldsymbol{T}\left(\boldsymbol{x}^{\prime}\right)\right] \mathrm{d} \boldsymbol{\xi} \mathrm{d} \boldsymbol{x} \mathrm{d} \boldsymbol{x}^{\prime} .
\end{aligned}
$$


Next we write out the matrix multiplications of (66) in summation form and use the linearity of the expectation operation. We obtain

$$
\begin{aligned}
\mathcal{J}_{T}\left(\underline{Q)} \approx \tilde{\mathcal{J}}_{T}(\underline{Q})\right. & +\mathcal{B}(\underline{Q}) \\
\sim & \int \mathrm{e}^{\mathrm{i}\left(\boldsymbol{x}-\boldsymbol{x}^{\prime}\right) \cdot \boldsymbol{\xi}} \operatorname{tr}\left[\left\{\underline{Q}\left(\boldsymbol{x}^{\prime}, \boldsymbol{\xi}\right) \underline{A}^{T}\left(\boldsymbol{x}^{\prime}, \boldsymbol{\xi}\right) \eta\left(\boldsymbol{x}^{\prime}, \boldsymbol{x}^{\prime}, \boldsymbol{\xi}\right)-\underline{\tilde{x}}_{\Omega}\left(\boldsymbol{x}^{\prime}, \boldsymbol{\xi}\right)\right\}^{\dagger}\right. \\
& \left.\times\left\{\underline{Q}\left(\boldsymbol{x}^{\prime}, \boldsymbol{\xi}\right) \underline{A}^{T}\left(\boldsymbol{x}^{\prime}, \boldsymbol{\xi}\right) \eta\left(\boldsymbol{x}^{\prime}, \boldsymbol{x}^{\prime}, \boldsymbol{\xi}\right)-\underline{\tilde{\chi}}_{\Omega}\left(\boldsymbol{x}^{\prime}, \boldsymbol{\xi}\right)\right\} \underline{C}^{T}\left(\boldsymbol{x}^{\prime}, \boldsymbol{x}\right)\right] \mathrm{d} \boldsymbol{\xi} \mathrm{d} \boldsymbol{x} \mathrm{d} \boldsymbol{x}^{\prime} \\
& +\int \mathrm{e}^{\mathrm{i}\left(\boldsymbol{x}-\boldsymbol{x}^{\prime}\right) \cdot \boldsymbol{\xi}} \operatorname{tr}\left[\left\{\underline{Q}\left(\boldsymbol{x}^{\prime}, \boldsymbol{\xi}\right) \underline{A}^{T}\left(\boldsymbol{x}^{\prime}, \boldsymbol{\xi}\right) \eta\left(\boldsymbol{x}^{\prime}, \boldsymbol{x}^{\prime}, \boldsymbol{\xi}\right)-\underline{x}_{\Omega}\left(\boldsymbol{x}^{\prime}, \boldsymbol{\xi}\right)\right\}^{\dagger}\right. \\
& \left.\times\left\{\underline{Q}\left(\boldsymbol{x}^{\prime}, \boldsymbol{\xi}\right) \underline{A}^{T}\left(\boldsymbol{x}^{\prime}, \boldsymbol{\xi}\right) \eta\left(\boldsymbol{x}^{\prime}, \boldsymbol{x}^{\prime}, \boldsymbol{\xi}\right)-\underline{\tilde{x}}_{\Omega}\left(\boldsymbol{x}^{\prime}, \boldsymbol{\xi}\right)\right\} \boldsymbol{\mu}\left(\boldsymbol{x}^{\prime}\right) \boldsymbol{\mu}^{\dagger}(\boldsymbol{x})\right] \mathrm{d} \boldsymbol{\xi} \mathrm{d} \boldsymbol{x} \mathrm{d} \boldsymbol{x}^{\prime},
\end{aligned}
$$

where $\operatorname{tr}(\cdot)$ denotes the trace of the matrix argument. The second integral of (67) is the bias term of the MSE. The remaining terms make up the variance portion.

We can repeat the same steps for the clutter term $\mathcal{J}_{C}(Q)$ to obtain the leading-order expression

$$
\begin{aligned}
\mathcal{J}_{C}(\underline{Q}) \approx \int \mathrm{e}^{\mathrm{i}\left(\boldsymbol{x}-\boldsymbol{x}^{\prime}\right) \cdot \boldsymbol{\xi}} \operatorname{tr}[ & \left\{\underline{Q}\left(\boldsymbol{x}^{\prime}, \boldsymbol{\xi}\right) \underline{A}^{C}\left(\boldsymbol{x}^{\prime}, \boldsymbol{\xi}\right) \eta\left(\boldsymbol{x}^{\prime}, \boldsymbol{x}^{\prime}, \boldsymbol{\xi}\right)\right\}^{\dagger} \\
& \left.\times\left\{\underline{Q}\left(\boldsymbol{x}^{\prime}, \boldsymbol{\xi}\right) \underline{A}^{C}\left(\boldsymbol{x}^{\prime}, \boldsymbol{\xi}\right) \eta\left(\boldsymbol{x}^{\prime}, \boldsymbol{x}^{\prime}, \boldsymbol{\xi}\right)\right\} \underline{\mathcal{R}}^{C}\left(\boldsymbol{x}^{\prime}, \boldsymbol{x}\right)\right] \mathrm{d} \boldsymbol{\xi} \mathrm{d} \boldsymbol{x} \mathrm{d} \boldsymbol{x}^{\prime} .
\end{aligned}
$$

The last term we need to simplify is the noise term, i.e. $\mathcal{J}_{n}(\underline{Q})$, which we write explicitly as

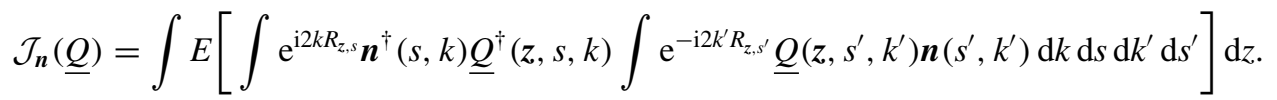

We rewrite the matrix and vector multiplication as before to obtain

$\mathcal{J}_{n}(\underline{Q})=\int \mathrm{e}^{\mathrm{i} 2\left(k R_{z, s}-k^{\prime} R_{z, s^{\prime}}\right)} \operatorname{tr}\left[\underline{Q}^{\dagger}(z, s, k) \underline{Q}\left(z, s^{\prime}, k^{\prime}\right) \underline{S}^{n}\left(s^{\prime}, k^{\prime} ; s, k\right)\right] \mathrm{d} k \mathrm{~d} s \mathrm{~d} k^{\prime} \mathrm{d} s^{\prime} \mathrm{d} z$,

where $S^{n}$ is the matrix of covariance functions of the noise. We denote it by the letter $S$ because the noise is already written in terms of a frequency variable $k$ and is therefore analogous to a spectral density function. In order to simplify our expression so that we may add it to the target and clutter terms, we make the assumption that the noise is stationary in both $s$ and $k$ :

$$
S_{i, j}^{n}\left(s, k ; s^{\prime}, k^{\prime}\right)=\tilde{S}_{i, j}^{n}(s, k) \delta\left(s-s^{\prime}\right) \delta\left(k-k^{\prime}\right) .
$$

This is equivalent to assuming that the noise has been prewhitened. Inserting (71) into equation (70), we obtain

$$
\mathcal{J}_{n}(\underline{Q})=\int \operatorname{tr}\left[\underline{Q}^{\dagger}(z, s, k) \underline{Q}(z, s, k) \underline{\tilde{S}}^{n}(s, k)\right] \mathrm{d} k \mathrm{~d} s \mathrm{~d} z
$$

where we have also dropped the primes on the dummy variables $s^{\prime}$ and $k^{\prime}$. Our last step is to perform the Stolt change of variables from $(s, k)$ to $\xi$ to obtain (51).

We now rewrite our expression for $\mathcal{J}_{T}$ and $\mathcal{J}_{C}$ in terms of the spatial frequency variable. We use the symmetry of covariances to rewrite (44) and (45) as

$$
\begin{aligned}
& \underline{C}^{T}\left(\boldsymbol{x}^{\prime}, \boldsymbol{x}\right)=\int \mathrm{e}^{-\mathrm{i} \boldsymbol{x}^{\prime} \cdot \boldsymbol{\zeta}} \mathrm{e}{ }^{\mathrm{i} x \cdot \boldsymbol{\zeta}^{\prime}} \underline{S}_{T}\left(\boldsymbol{\zeta}, \boldsymbol{\zeta}^{\prime}\right) \mathrm{d} \boldsymbol{\zeta} \mathrm{d} \boldsymbol{\zeta}^{\prime} \\
& \underline{\mathcal{R}}^{C}\left(\boldsymbol{x}^{\prime}, \boldsymbol{x}\right)=\int \mathrm{e}^{-\mathrm{i} \boldsymbol{x}^{\prime} \cdot \boldsymbol{\zeta}} \mathrm{e}^{\mathrm{i} x \cdot \boldsymbol{\zeta}^{\prime}} \underline{S}_{C}\left(\boldsymbol{\zeta}, \boldsymbol{\zeta}^{\prime}\right) \mathrm{d} \boldsymbol{\zeta} \mathrm{d} \boldsymbol{\zeta}^{\prime}
\end{aligned}
$$

which we then insert into (67) and (68):

$$
\begin{aligned}
\tilde{\mathcal{J}}_{T}\left(\underline { Q ) } \approx \int \mathrm { e } ^ { \mathrm { i } ( \boldsymbol { x } - \boldsymbol { x } ^ { \prime } ) \cdot \boldsymbol { \xi } } \mathrm { e } ^ { - \mathrm { i } ( \boldsymbol { x } ^ { \prime } \cdot \zeta - \boldsymbol { x } \cdot \boldsymbol { \zeta } ^ { \prime } ) } \operatorname { t r } \left[\left\{\underline{Q}\left(\boldsymbol{x}^{\prime}, \boldsymbol{\xi}\right) \underline{A}^{T}\left(\boldsymbol{x}^{\prime}, \boldsymbol{\xi}\right) \eta\left(\boldsymbol{x}^{\prime}, \boldsymbol{x}^{\prime}, \boldsymbol{\xi}\right)-\underline{\tilde{x}}_{\Omega}\left(\boldsymbol{x}^{\prime}, \boldsymbol{\xi}\right)\right\}^{\dagger}\right.\right. \\
\left.\quad \times\left\{\underline{Q}\left(\boldsymbol{x}^{\prime}, \boldsymbol{\xi}\right) \underline{A}^{T}\left(\boldsymbol{x}^{\prime}, \boldsymbol{\xi}\right) \eta\left(\boldsymbol{x}^{\prime}, \boldsymbol{x}^{\prime}, \boldsymbol{\xi}\right)-\underline{\tilde{\chi}}_{\Omega}\left(\boldsymbol{x}^{\prime}, \boldsymbol{\xi}\right)\right\} \underline{S}_{T}\left(\boldsymbol{\zeta}, \boldsymbol{\zeta}^{\prime}\right)\right] \mathrm{d} \boldsymbol{\xi} \mathrm{d} \boldsymbol{x} \mathrm{d} \boldsymbol{x}^{\prime} \mathrm{d} \boldsymbol{\zeta} \mathrm{d} \boldsymbol{\zeta}^{\prime}
\end{aligned}
$$


and

$$
\begin{aligned}
\mathcal{J}_{C}(\underline{Q}) \approx \int \mathrm{e}^{\mathrm{i}\left(\boldsymbol{x}-\boldsymbol{x}^{\prime}\right) \cdot \xi} \mathrm{e}^{-\mathrm{i}\left(\boldsymbol{x}^{\prime} \cdot \xi-\boldsymbol{x} \cdot \boldsymbol{\zeta}^{\prime}\right)} \operatorname{tr}\left[\left\{\underline{Q}\left(\boldsymbol{x}^{\prime}, \boldsymbol{\xi}\right) \underline{A}^{C}\left(\boldsymbol{x}^{\prime}, \boldsymbol{\xi}\right) \eta\left(\boldsymbol{x}^{\prime}, \boldsymbol{x}^{\prime}, \boldsymbol{\xi}\right)\right\}^{\dagger}\right. \\
\left.\quad \times\left\{\underline{Q}\left(\boldsymbol{x}^{\prime}, \boldsymbol{\xi}\right) \underline{A}^{C}\left(\boldsymbol{x}^{\prime}, \boldsymbol{\xi}\right) \eta\left(\boldsymbol{x}^{\prime}, \boldsymbol{x}^{\prime}, \boldsymbol{\xi}\right)\right\} \underline{S}_{C}\left(\boldsymbol{\zeta}, \boldsymbol{\zeta}^{\prime}\right)\right] \mathrm{d} \boldsymbol{\xi} \mathrm{d} \boldsymbol{x} \mathrm{d} \boldsymbol{x}^{\prime} \mathrm{d} \boldsymbol{\zeta} \mathrm{d} \boldsymbol{\zeta}^{\prime} .
\end{aligned}
$$

In (74) and (75), we carry out first the $\boldsymbol{x}$ integration, which results in $\delta\left(\boldsymbol{\zeta}^{\prime}-\xi\right)$, and then the $\boldsymbol{\xi}$ integration to obtain (48) and (49).

To address the bias term, $\mathcal{B}(\underline{Q})$, we introduce the function $\underline{M}$, which is analogous to a spectral density function and is defined as in equation (46). We substitute (46) with the arguments reversed into the expression for $\mathcal{B}(\underline{Q})$ and perform the same symbol calculations to arrive at (50).

\section{Theorem.}

(1) Under the same assumptions as the above lemma, any filter $Q$ satisfying a symbol estimate and also minimizing the leading-order $\operatorname{MSE} \mathcal{J}(\underline{Q})$ must be a solution of the following integral equation $\forall r$ and $\forall q$ :

$$
\left(\int \bar{\eta} \mathrm{e}^{\mathrm{i} x \cdot\left(\zeta^{\prime}-\zeta\right)}\left[\left(\underline{Q}^{T} \eta-\underline{\tilde{x}}_{\Omega}\right)\left(\underline{S}_{T}+\underline{M}\right)\left(\underline{A}^{T}\right)^{\dagger}+\left(\underline{Q}^{C} \eta\right) \underline{S}_{C}\left(\underline{A}^{C}\right)^{\dagger}\right] \mathrm{d} \zeta^{\prime}\right)_{(r, q)}+\left(\underline{Q} \underline{\tilde{S}}^{n} \eta\right)_{(r, q)}=0 .
$$

Here 'leading order' is in the microlocal sense [15, 33], meaning that the higher order terms are smoother than the leading-order one.

(2) If, in addition, we make the stationarity assumptions

$$
\begin{aligned}
& \underline{S}_{T}\left(\zeta, \zeta^{\prime}\right)=\underline{S}_{T}(\zeta) \delta\left(\zeta-\zeta^{\prime}\right), \\
& \underline{S}_{C}\left(\zeta, \zeta^{\prime}\right)=\underline{S}_{C}(\zeta) \delta\left(\zeta-\zeta^{\prime}\right),
\end{aligned}
$$

then the filter $\underline{Q}$ minimizing the leading-order total error variance $\mathcal{V}(\underline{Q})$ is given by

$$
\underline{Q}^{\dagger}=\left[|\eta|^{2}\left(\underline{A}^{T}\left(\underline{S}_{T}\right)^{\dagger}\left(\underline{A}^{T}\right)^{\dagger}+\underline{A}^{C}\left(\underline{S}^{C}\right)^{\dagger}\left(\underline{A}^{C}\right)^{\dagger}\right)+\eta\left(\tilde{S}^{n}\right)^{\dagger}\right]^{-1} \eta \underline{A}^{T}\left(\underline{S}_{T}\right)^{\dagger} \underline{\tilde{x}}_{\Omega}^{\dagger}
$$

if the matrix in brackets is indeed invertible.

Part (2) of this theorem is included because it results in a simpler algebraic expression for $Q$, which aids in numerical calculations.

Proof. In order to find the $\underline{Q}$ which minimizes $\mathcal{J}(\underline{Q})$, we take the variation of $\mathcal{J}$ with respect to $Q$. That is, we look for the $Q$ which satisfies

$$
\begin{aligned}
0=\left.\frac{\mathrm{d}}{\mathrm{d} \epsilon}\right|_{\epsilon=0} \tilde{\mathcal{J}}_{T}\left(\underline{Q}+\epsilon \underline{Q}_{\epsilon}\right)+\left.\frac{\mathrm{d}}{\mathrm{d} \epsilon}\right|_{\epsilon=0} \mathcal{J}_{C}\left(\underline{Q}+\epsilon \underline{Q}_{\epsilon}\right)+\left.\frac{\mathrm{d}}{\mathrm{d} \epsilon}\right|_{\epsilon=0} \mathcal{J}_{n}\left(\underline{Q}+\epsilon \underline{Q}_{\epsilon}\right) \\
+\left.\frac{\mathrm{d}}{\mathrm{d} \epsilon}\right|_{\epsilon=0} \mathcal{B}\left(\underline{Q}+\epsilon \underline{Q}_{\epsilon}\right)
\end{aligned}
$$

for all possible $\underline{Q}$.

To calculate this derivative, we focus on the first term on the right-hand side of (80) and then apply similar steps to obtain the other terms in the derivative. Applying the product rule to (48), we have

$$
\begin{aligned}
\left.\frac{\mathrm{d}}{\mathrm{d} \epsilon}\right|_{\epsilon=0} \tilde{\mathcal{J}}_{T}\left(\underline{Q}+\epsilon \underline{Q}_{\epsilon}\right)=\int & \mathrm{e}^{\mathrm{i} x \cdot\left(\zeta^{\prime}-\zeta\right)} \operatorname{tr}\left[\left(\underline{Q}_{\epsilon} \underline{A}^{T} \eta\right)^{\dagger}\left(\left(\underline{Q}^{T} \underline{T}^{T}-\underline{\tilde{\chi}}_{\Omega}\right) \underline{S}_{T}\right)\right] \mathrm{d} \boldsymbol{x} \mathrm{d} \zeta \mathrm{d} \zeta^{\prime} \\
& +\int \mathrm{e}^{\mathrm{i} x \cdot\left(\zeta^{\prime}-\zeta\right)} \operatorname{tr}\left[\left(\underline{Q}^{T} \underline{A}^{T} \eta-\underline{\tilde{x}}_{\Omega}\right)^{\dagger}\left(\left(\underline{Q}_{\epsilon} \underline{A}^{T} \eta\right) \underline{S}_{T}\right)\right] \mathrm{d} \boldsymbol{x} \mathrm{d} \zeta \mathrm{d} \zeta^{\prime} .
\end{aligned}
$$


Now if we interchange $\zeta$ and $\zeta^{\prime}$ in the second integral and use the fact that $\underline{S}_{T}^{\dagger}\left(\zeta, \zeta^{\prime}\right)=\underline{S}_{T}\left(\zeta^{\prime}, \zeta\right)$, then we obtain

$$
\begin{aligned}
\left.\frac{\mathrm{d}}{\mathrm{d} \epsilon}\right|_{\epsilon=0} \tilde{\mathcal{J}}_{T}\left(\underline{Q}+\epsilon \underline{Q}_{\epsilon}\right)= & \int \mathrm{e}^{\mathrm{i} x \cdot\left(\zeta^{\prime}-\zeta\right)} \operatorname{tr}\left[\left(\underline{Q}_{\epsilon} \underline{A}^{T} \eta\right)^{\dagger}\left(\left(\underline{Q} \underline{A}^{T} \eta-\underline{\tilde{x}}_{\Omega}\right) \underline{S}_{T}\right)\left(\boldsymbol{\zeta}, \boldsymbol{\zeta}^{\prime}\right)\right] \mathrm{d} \boldsymbol{x} \mathrm{d} \zeta \mathrm{d} \boldsymbol{\zeta}^{\prime} \\
& +\int \mathrm{e}^{\mathrm{i} \boldsymbol{x} \cdot\left(\zeta-\zeta^{\prime}\right)} \operatorname{tr}\left[\left(\underline{Q}^{A} \underline{A}^{T} \eta-\underline{\tilde{\chi}}_{\Omega}\right)^{\dagger}\left(\left(\underline{Q}_{\epsilon} \underline{A}^{T} \eta\right) \underline{S}_{T}^{\dagger}\left(\boldsymbol{\zeta}, \boldsymbol{\zeta}^{\prime}\right)\right)\right] \mathrm{d} \boldsymbol{x} \mathrm{d} \zeta \mathrm{d} \boldsymbol{\zeta}^{\prime} .
\end{aligned}
$$

Next we use the fact that for any square matrix $\underline{\mathcal{M}}, \operatorname{tr}(\underline{\mathcal{M}})=\operatorname{tr}\left(\underline{\mathcal{M}}^{\prime}\right)$ (where the superscript ' here refers to transpose) and the fact that for any square matrices $\underline{A}, \underline{B}$ and $\underline{C}, \operatorname{tr}(\underline{A B C})=\operatorname{tr}(\underline{B C A})$ to obtain

$$
\begin{aligned}
\left.\frac{\mathrm{d}}{\mathrm{d} \epsilon}\right|_{\epsilon=0} \tilde{\mathcal{J}}_{T}\left(\underline{Q}+\epsilon \underline{Q}_{\epsilon}\right)= & \int \mathrm{e}^{\mathrm{i} x \cdot\left(\zeta^{\prime}-\zeta\right)} \operatorname{tr}\left[\left(\underline{Q}_{\epsilon} \underline{A}^{T} \eta\right)^{\dagger}\left(\left(\underline{Q A^{T}} \eta-\underline{\tilde{\chi}}_{\Omega}\right) \underline{S}_{T}\right)\left(\zeta, \zeta^{\prime}\right)\right] \mathrm{d} \boldsymbol{x} \mathrm{d} \zeta \mathrm{d} \zeta^{\prime} \\
& \left.+\int \mathrm{e}^{\mathrm{i} \boldsymbol{x} \cdot\left(\zeta-\zeta^{\prime}\right)} \operatorname{tr}\left[\left(\underline{Q}_{\epsilon} \underline{A}^{T} \eta\right)^{\prime} \overline{\left(\underline{Q}^{T}\right.} \eta-\underline{\tilde{\chi}}_{\Omega}\right) \underline{S}_{T}\left(\zeta, \boldsymbol{\zeta}^{\prime}\right)\right] \mathrm{d} \boldsymbol{x} \mathrm{d} \zeta \mathrm{d} \zeta^{\prime} .
\end{aligned}
$$

We note that the second term is exactly the complex conjugate of the first term. This leads us to the following expression:

$\left.\frac{\mathrm{d}}{\mathrm{d} \epsilon}\right|_{\epsilon=0} \tilde{\mathcal{J}}_{T}\left(\underline{Q}+\epsilon \underline{Q}_{\epsilon}\right)=2 \operatorname{Re} \int \mathrm{e}^{\mathrm{i} x \cdot\left(\zeta^{\prime}-\zeta\right)} \operatorname{tr}\left[\left(\underline{Q}_{\epsilon} \underline{A}^{T} \eta\right)^{\dagger}\left(\left(\underline{Q} \underline{A}^{T} \eta-\underline{\tilde{\chi}}_{\Omega}\right) \underline{S}_{T}\right)\right] \mathrm{d} \boldsymbol{x} \mathrm{d} \zeta \mathrm{d} \zeta^{\prime}$.

Performing similar steps, we obtain the expressions for the variational derivatives of $\mathcal{J}_{C}, \mathcal{J}_{n}$ and $\mathcal{B}$ :

$\left.\frac{\mathrm{d}}{\mathrm{d} \epsilon}\right|_{\epsilon=0} \mathcal{J}_{C}\left(\underline{Q}+\epsilon \underline{Q}_{\epsilon}\right)=2 \operatorname{Re} \int \mathrm{e}^{\mathrm{i} x \cdot\left(\zeta^{\prime}-\zeta\right)} \operatorname{tr}\left[\left(\underline{Q}_{\epsilon} \underline{A}^{C} \eta\right)^{\dagger}\left(\left(\underline{Q}^{C} \underline{A}^{C} \eta \underline{S}_{C}\right)\right] \mathrm{d} \boldsymbol{x} \mathrm{d} \zeta \mathrm{d} \zeta^{\prime}\right.$,

$\left.\frac{\mathrm{d}}{\mathrm{d} \epsilon}\right|_{\epsilon=0} \mathcal{J}_{n}\left(\underline{Q}+\epsilon \underline{Q}_{\epsilon}\right)=2 \operatorname{Re} \int \operatorname{tr}\left[\underline{Q}_{\epsilon}^{\dagger} \underline{Q}^{n}\right] \eta \mathrm{d} \boldsymbol{x} \mathrm{d} \zeta$

$\left.\frac{\mathrm{d}}{\mathrm{d} \epsilon}\right|_{\epsilon=0} \mathcal{B}\left(\underline{Q}+\epsilon \underline{Q}_{\epsilon}\right)=2 \operatorname{Re} \int \mathrm{e}^{\mathrm{i} x \cdot\left(\zeta^{\prime}-\zeta\right)} \operatorname{tr}\left[\left(\underline{Q}_{\epsilon} \underline{A}^{T} \eta\right)^{\dagger}\left(\left(\underline{Q}^{A} \underline{A}^{T} \eta-\underline{\tilde{x}}_{\Omega}\right) \underline{M}\right)\right] \mathrm{d} \boldsymbol{x} \mathrm{d} \zeta \mathrm{d} \zeta^{\prime}$.

Now inserting the above results into equation (80) we have

$$
\begin{aligned}
0=2 \operatorname{Re} \int \mathrm{e}^{\mathrm{i} x \cdot\left(\zeta^{\prime}-\zeta\right)} \operatorname{tr}\left[\left(\underline{Q}_{\epsilon} \underline{A}^{T} \eta\right)^{\dagger}\left(\left(\underline{Q}_{A^{T}} \eta-\underline{\tilde{\chi}}_{\Omega}\right)\left(\underline{S}_{T}+\underline{M}\right)\right)\right] \mathrm{d} \boldsymbol{x} \mathrm{d} \zeta \mathrm{d} \zeta^{\prime} \\
+2 \operatorname{Re} \int \mathrm{e}^{\mathrm{i} x \cdot\left(\zeta^{\prime}-\zeta\right)} \operatorname{tr}\left[\left(\underline{Q}_{\epsilon} \underline{A}^{C} \eta\right)^{\dagger}\left(\left(\underline{Q A^{C}} \eta\right) \underline{S}_{C}\right)\right] \mathrm{d} \boldsymbol{x} \mathrm{d} \zeta \mathrm{d} \zeta^{\prime} \\
+2 \operatorname{Re} \int \operatorname{tr}\left[\underline{Q}_{\epsilon}^{\dagger} \underline{Q} \underline{\tilde{S}^{n}}\right] \eta \mathrm{d} \boldsymbol{x} \mathrm{d} \zeta .
\end{aligned}
$$

In the first two terms we use the fact that for any square matrices $\underline{A}, \underline{B}, \underline{C}$ and $\underline{D}$, we have $\operatorname{tr}(\underline{A B C D})=\operatorname{tr}(\underline{B C D A})$. This allows us to write

$$
\begin{aligned}
0=2 \operatorname{Re} \int \operatorname{tr}\left[\underline { Q } _ { \epsilon } ^ { \dagger } \left\{\hat { \overline { \eta } } \mathrm { e } ^ { \mathrm { i } x \cdot ( \zeta ^ { \prime } - \zeta ) } \left[\left(\underline{Q}^{A^{T}} \eta-\underline{\tilde{\chi}}_{\Omega}\right)\left(\underline{S}_{T}+\underline{M}\right)\left(\underline{A}^{T}\right)^{\dagger}\right.\right.\right. \\
\left.\left.\left.+\left(\underline{Q} \underline{A}^{C} \eta\right)\left(\underline{S}_{C}\right)\left(\underline{A}^{C}\right)^{\dagger}\right]\right\}\right] \mathrm{d} \boldsymbol{x} \mathrm{d} \zeta \mathrm{d} \zeta^{\prime}+2 \operatorname{Re} \int \operatorname{tr}\left[\underline{Q}_{\epsilon}^{\dagger} \underline{Q} \underline{\tilde{S}} \underline{\tilde{N}}^{n}\right] \mathrm{d} \boldsymbol{x} \mathrm{d} \zeta .
\end{aligned}
$$

Next we write out the trace operator in the summation form:

$$
\begin{gathered}
0=2 \operatorname{Re} \int \sum_{q=1}^{4} \sum_{r=1}^{4} Q_{\epsilon,(q, r)}^{\dagger}\left\{\int \overline { \eta } \mathrm { e } ^ { \mathrm { i } x \cdot ( \zeta ^ { \prime } - \zeta ) } \left[\left(\underline{Q}^{T} \underline{\underline{\chi}}^{T} \eta \underline{\tilde{x}}_{\Omega}\right)\left(\underline{S}_{T}+\underline{M}\right)\left(\underline{A}^{T}\right)^{\dagger}\right.\right. \\
\left.\left.+\left(\underline{Q}^{C} \eta\right)\left(\underline{S}_{C}\right)\left(\underline{A}^{C}\right)^{\dagger}\right]_{(r, q)} \mathrm{d} \zeta^{\prime}+\left(\underline{Q}_{\underline{S}}^{n} \eta\right)_{(r, q)}\right\} \mathrm{d} \boldsymbol{x} \mathrm{d} \zeta .
\end{gathered}
$$


We see that since $\underline{Q} \epsilon$ is arbitrary in (88), $\underline{Q}$ must satisfy the integral equation (76). This completes the proof of part (1) of the theorem.

To prove part (2) of the theorem, we minimize the variance of the error process. We begin with the leading-order contribution to $\mathcal{V}(\underline{Q})$ (the variance of the error term), which is given by

$$
\mathcal{V}(\underline{Q})=\tilde{\mathcal{J}}_{T}(\underline{Q})+\mathcal{J}_{C}(\underline{Q})+\mathcal{J}_{n}(\underline{Q}) .
$$

Note the only term missing is the bias-related term $\mathcal{B}(Q)$.

Following the above calculations, we find that for all $r$ and $q$,

$0=\left(\int \bar{\eta} \mathrm{e}^{\mathrm{i} x \cdot\left(\zeta^{\prime}-\zeta\right)}\left[\left(\underline{Q} \underline{A}^{T} \eta-\underline{\tilde{x}}_{\Omega}\right) \underline{S}_{T}\left(\underline{A}^{T}\right)^{\dagger}+\left(\underline{Q} \underline{A}^{C} \eta\right) \underline{S}_{C}\left(\underline{A}^{C}\right)^{\dagger}\right] \mathrm{d} \zeta^{\prime}\right)_{(r, q)}+\left(\underline{Q} \underline{\tilde{S}}^{n} \eta\right)_{(r, q)}$.

With the stationarity assumptions (77), we obtain

$$
\underline{Q}\left[|\eta|^{2}\left(\underline{A}^{T} \underline{S}_{T}\left(\underline{A}^{T}\right)^{\dagger}+\underline{A}^{C} \underline{S}_{C}\left(\underline{A}^{C}\right)^{\dagger}\right)+\eta \underline{\tilde{S}^{n}}\right]=\bar{\eta} \underline{\tilde{x}}_{\Omega} \underline{S}_{T}\left(\underline{A}^{T}\right)^{\dagger}
$$

from which we obtain (79). This completes the proof of the second part of the theorem.

\section{Numerical experiments}

We performed numerical experiments to verify our theory. The scene on the ground is assumed to be $50 \mathrm{~m} \times 50 \mathrm{~m}$, represented by $100 \times 100$ pixels. That is, our resolution cell size is $0.5 \mathrm{~m} \times 0.5 \mathrm{~m}$. The endpoints of the scene are $( \pm 25, \pm 25)(\mathrm{m})$. We consider targets with fixed orientation and we assume that $\rho_{T}(\boldsymbol{x})=1$ for all target locations $\boldsymbol{x}$. In addition, we assume the target is always 20 pixels in length and 1 pixel in width. Thus the target is deterministic for our simulations; we leave stochastic target simulations as future work. For the clutter process, we assume that a clutter dipole is located at every position $\boldsymbol{x}$ in the scene of interest. The values of the clutter scattering strength for all ground locations, $\rho_{C}(\boldsymbol{x})$, are independent identically distributed (i.i.d.) Gaussian random variables with zero mean and unit complex variance. In addition, we assume that the orientations of the clutter dipoles, $\theta_{C}(\boldsymbol{x})$, are i.i.d. uniform random variables between the angles $[0, \pi / 2]$. We note that in this case the total clutter process $\boldsymbol{C}(\boldsymbol{x})$ is wide-sense stationary and therefore the stationarity assumption (77) holds. Measurement noise is not explicitly included in the numerical simulations; this is equivalent to assuming that the data have been prewhitened.

The flight path is a linear one along the $y$-axis; we take $\boldsymbol{\gamma}(s)=\left[x_{0}, s, z_{0}\right]$, where $-30 \leqslant s \leqslant 30$ (in meters) and we have assumed that $x_{0}=20 \mathrm{~m}$ and $z_{0}=10 \mathrm{~m}$ are fixed. The two antennas used for transmission and reception have orientations $\widehat{\boldsymbol{e}}_{a}=[1,0,0]^{\prime}$ and $\widehat{\boldsymbol{e}}_{b}=[0,1,0]^{\prime}$, which are defined with respect to the origin in the scene on the ground. We may think of $a$ as having the vertical or $V$ orientation, and $b$ as having the horizontal or $H$ orientation. Our frequency range is $1-1.5 \mathrm{GHz}$, where we sample at a rate above Nyquist.

Since the target vector field is deterministic, we estimate its spectral density function via the formula

$$
\underline{S}_{T}(\zeta)=\left|\int \mathrm{e}^{-\mathrm{ix} \cdot \zeta} \boldsymbol{T}(\boldsymbol{x}) \mathrm{d} \boldsymbol{x}\right|^{2} .
$$

The clutter covariance matrix was calculated by averaging over $\boldsymbol{C}(\boldsymbol{x})$ and $\boldsymbol{C}\left(\boldsymbol{x}^{\prime}\right)$ given the simple assumptions on its distribution. We then take the Fourier transform in order to calculate $\underline{S}_{C}(\zeta)$. Our definition of SCR is given by

$$
\mathrm{SCR}=20 \log \frac{\frac{1}{N} \sum_{1}^{N} \mid\left(\boldsymbol{T}\left(\boldsymbol{x}_{i}\right)-\left.\boldsymbol{\mu}_{T}\left(\boldsymbol{x}_{i}\right)\right|^{2}\right.}{E\left[|\boldsymbol{C}|^{2}\right]},
$$

where $N$ is the number of grid points and $\boldsymbol{\mu}_{T}$ is the mean of the target vector field. 

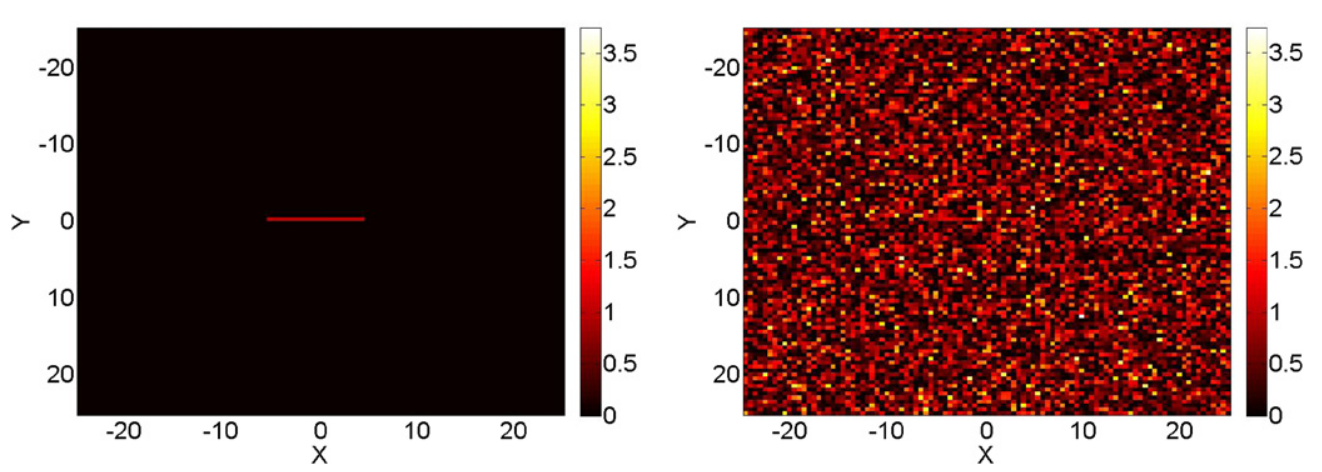

Figure 2. VV component of the target vector and target-plus-clutter vector, vertically polarized target.

In producing the data, we did not use the directional scattering assumptions on the target and clutter process (20) and (26). We instead used equation (11) to simulate the data produced by each transmitting and receiving antenna pair. That is, we do not approximate the radiation patterns when simulating the data. In this way, we avoid some of the corresponding 'inverse crimes' [9]. From the simulated data, we form images of the target vector field $\boldsymbol{T}(\boldsymbol{x})$ using equation (37) with the appropriate filters for the standard SAR image formation and our coupled polarimetric reconstruction. In the standard reconstruction, we assume that the transmitted field's polarization properties remain constant along the flight path and therefore perform standard backprojection on each element of the data vector. That is, we assume that the antenna polarization states and also the transmitted field's polarization states are given by $\widehat{\boldsymbol{e}}_{a}$ and $\widehat{\boldsymbol{e}}_{b}$ for all slow-time values instead of the true slow-time varying polarization state given in equation (17). We then utilize the resulting filter given in equation (91) (which is diagonal due to our assumption). For the coupled polarimetric reconstruction we allow the transmitted field's polarization states to vary according to equation (17) and again use the resulting filter $Q$ from equation (91) (which is full in this case). We then compare the two sets of images, $\overline{\boldsymbol{I}_{s}}(\boldsymbol{z})$ and $\boldsymbol{I}_{c}(\boldsymbol{z})$, resulting from the component-by-component backprojection and the coupled backprojection reconstruction methods, respectively. We also provide plots of the MSE versus the data SCR for both methods and tables demonstrating the image SCR ratios for the various cases.

Before we go into specific results, we note one issue present in our coupled numerical reconstruction scheme. The matrix in square brackets in equation (79) is typically close to being singular. To find its inverse numerically, we implemented a regularization scheme in which we diagonally weight the matrix in order to improve its condition number. This diagonal weighting depends on a constant factor, equivalent to the noise power, which constitutes our regularization parameter. The choice of the regularization parameter is done for each case individually and has not yet been optimized for minimizing MSE or maximizing image SCR. This is left for future work.

Example 1: vertically polarized target. We first consider the case when each sub-element (or individual target dipole) making up the extended target has the orientation $\widehat{\boldsymbol{e}}_{T}(\boldsymbol{x})=[1,0,0]^{\prime}$ which is parallel to the $a$, or $\mathrm{V}$, antenna. In figure 2, we show the actual target scene on the left and then the target-embedded-in-clutter scene on the right.

In figures 3 and 4 , we display the data obtained using the $a$ antenna for both transmission and reception, using $a$ for transmission and $b$ for reception, and also the case when $b$ is used 

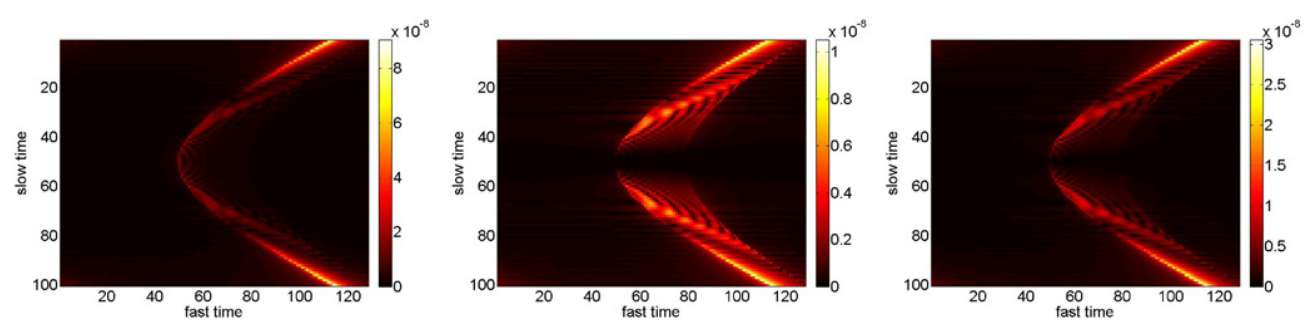

Figure 3. VV, HH and HV target-only data, vertically polarized target.
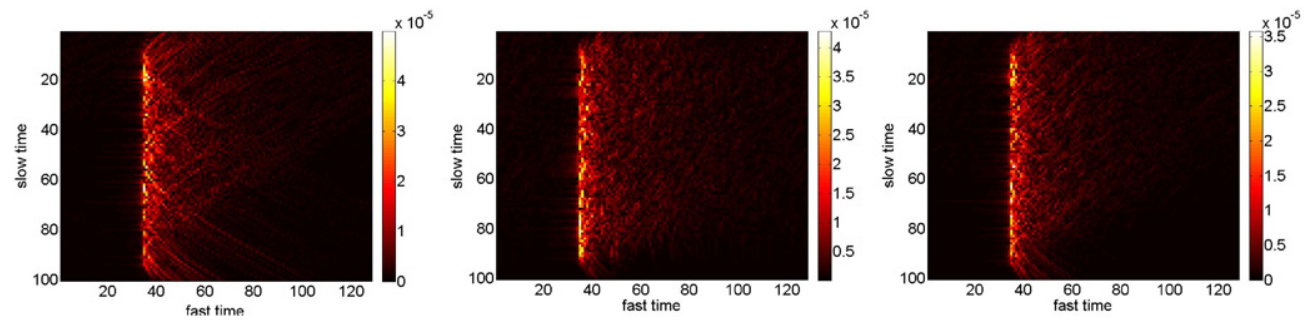

Figure 4. VV, HH and HV target-embedded-in-clutter data, vertically polarized target.
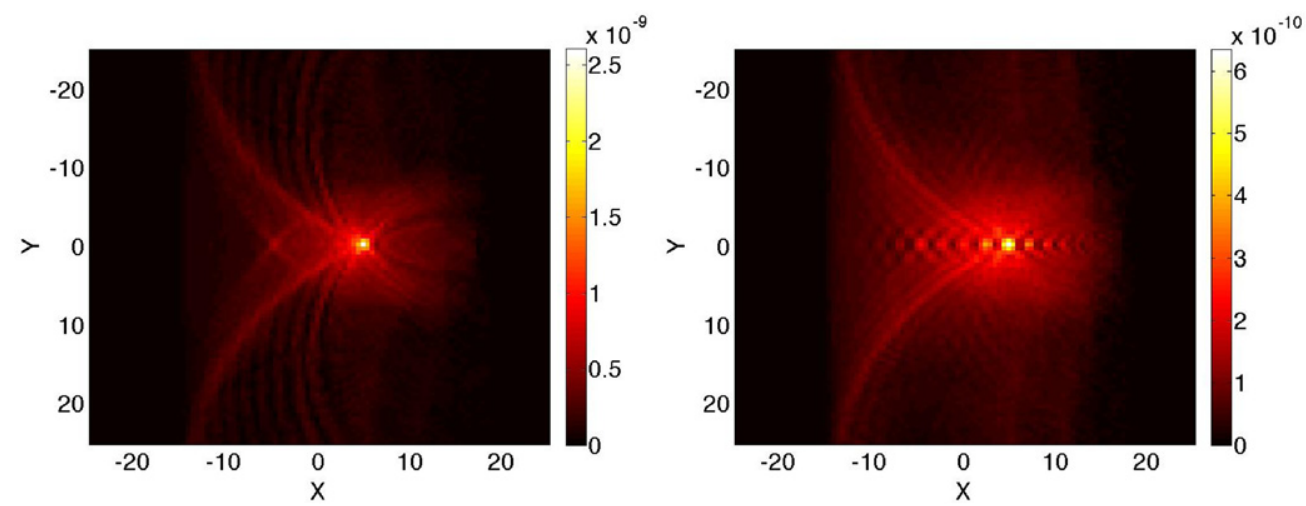

Figure 5. VV image created using the standard (left) and coupled (right) reconstructions.

for both processes. Note we do not display the data when using $b$ for transmission and $a$ for reception as in many practical situations this is identical to the $a$ transmit, $b$ receive case. Figure 3 shows target-only data, and figure 4 shows the data for the target embedded in clutter. As expected, there is no target response in the $\mathrm{HH}$ and $\mathrm{HV}$ channels when the antenna reaches the line $y=0(s=50)$, as this is the point where the flight path crosses the $x$-axis where the target lies, and consequently the target is viewed end-on. However there are data at this point in the VV data set, because the target orientation is parallel to the $\mathrm{V}$ antenna and the dot products in the data therefore have value 1 which is the maximum. When clutter is present, the target data are completely obscured.

Figure 5 shows the results of the standard image formation and the results of our coupled reconstruction. Here we only show the result of the VV image as the other two images are zero, as expected. In this case, the image SCR is $20 \mathrm{~dB}$. We note that the length of the target is more evident in the coupled reconstruction. This effect is due in part to the fact that the target 


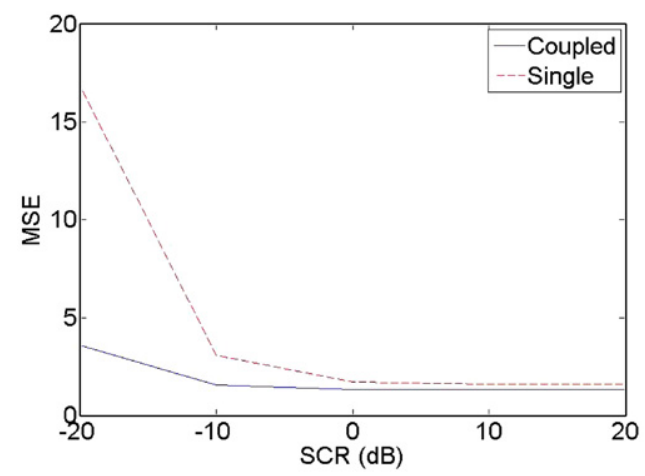

Figure 6. SCR versus MSE for the standard reconstructed images and coupled reconstructed images, respectively, vertically polarized target.

Table 1. Scene SCR in $\mathrm{dB}$ and standard versus coupled image SCR in dB, vertically polarized target.

\begin{tabular}{rcc}
\hline Scene SCR & Image SCR (standard) & Image SCR (coupled) \\
\hline-20 & 0.3881 & 0.2695 \\
-10 & 1.2272 & 0.8522 \\
0 & 3.8809 & 2.6949 \\
10 & 12.2723 & 8.5219 \\
20 & 38.8085 & 26.9486 \\
\hline
\end{tabular}

is visible in the data over a range of aspects; in other words, assumption (20) was not used in producing the simulated data.

We plot the SCR versus the MSE in figure 6. Observe that the coupled reconstruction performs significantly better when the SCR is small as is the case in more realisitc scenarios.

Lastly, we display the image SCR in table 1 . We calculate image SCR by performing the reconstruction techniques on target-only data and clutter-only data and then compare the energy in each set of images. We note that our coupled reconstruction does not provide improved image SCR in this example. This is due to the reduced intensity of the coupled images and also due to the $\mathrm{HH}$ and HV elements of the scattering vector being zero in this case. Thus the corresponding elements of the target spectral density matrix are zero as well. We therefore infer that using all data sets to reconstruct the VV scattering vector element does not increase the signal strength in this case.

Example 2: $45^{\circ}$ polarized target Our second example considers the case when each subelement of the extended target has orientation $\widehat{\boldsymbol{e}}_{T}=[1 / \sqrt{2}, 1 / \sqrt{2}, 0]^{\prime}$. We display the HV component of the target and the target-embedded-in-clutter scenes in figure 7 . In this case, we expect the coupled technique to aid in reconstructing the target more than in the previous example. In particular, the dot product of the transmitted field polarization state and target orientation reaches its maximal value during the course of the antenna trajectory. Therefore our model (i.e. the directional scattering assumption) is valid in this case. Also we note the elements of the spectral density matrix will all be of roughly the same magnitude because each element of the target scattering vector is of the same order. Therefore, we expect using all three data sets (shown in figure 8) to reconstruct each element of the scattering vector would 

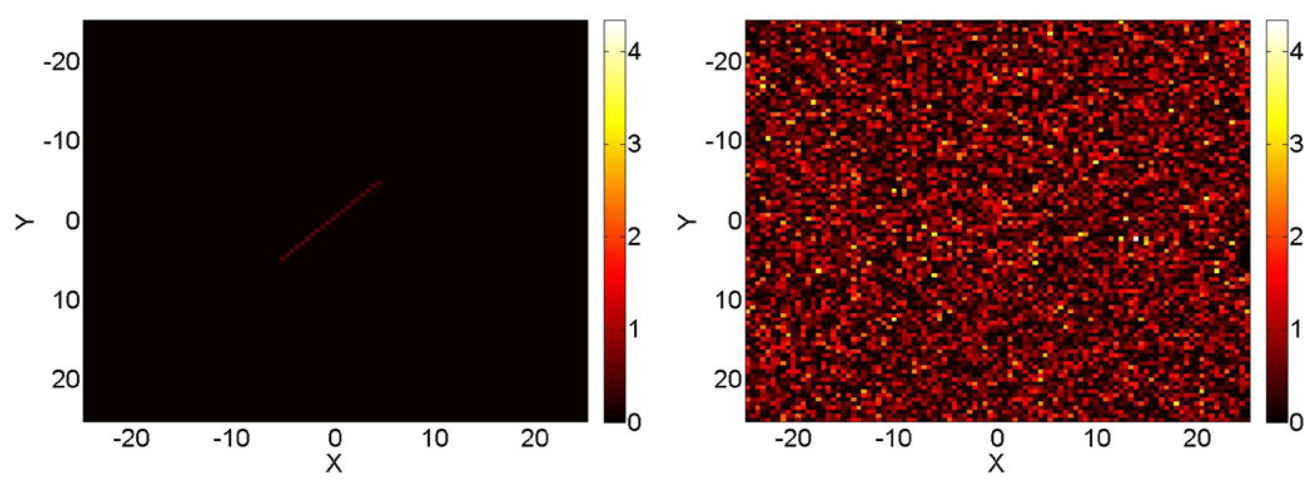

Figure 7. HV component of target vector and target plus clutter vector, $45^{\circ}$ polarized target.
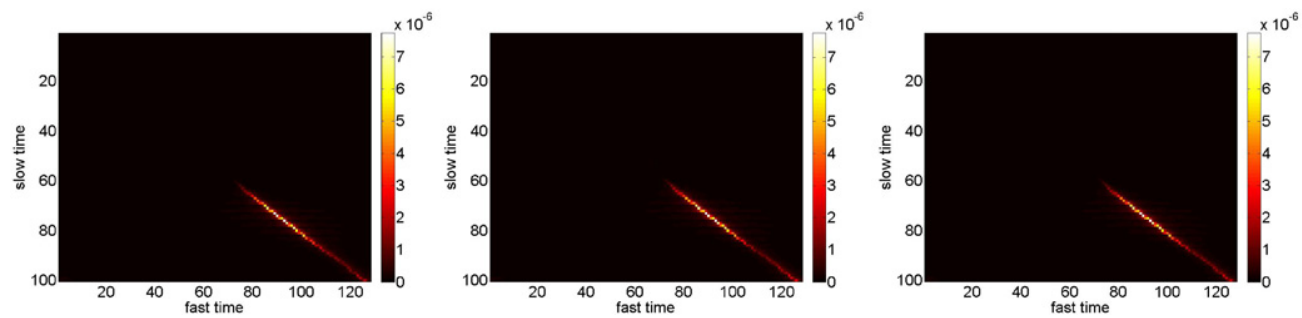

Figure 8. VV, $\mathrm{HH}$ and $\mathrm{HV}$ target only data, $45^{\circ}$ polarized target.
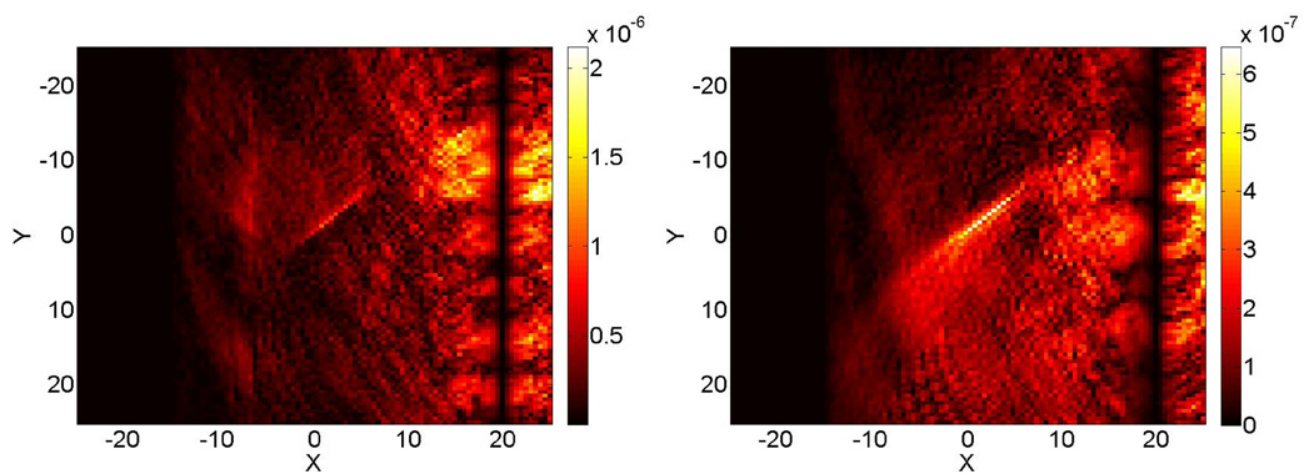

Figure 9. HV image created using the standard (left) and coupled (right) reconstructions, $45^{\circ}$ polarized target.

provide better images (i.e. lower MSE and higher image SCR) than the images formed using channel-by-channel backprojection.

In figure 9, we display example images formed using the two different techniques. Here we show only the result of the HV image as the other two images are almost identical. In this case, we have SCR of $-20 \mathrm{~dB}$. Note that the target is visible in both images, however the target stands out from the clutter significantly more in the image formed with our coupled technique. We see this effect reflected in table 2 where we calculate the image SCR for each technique. The SCR of the images formed using our technique is three times that of the images formed using the standard channel-by-channel backprojection. In addition, we see that the MSE is reduced as well in figure 10. The impact is particularly visible when the SCR of the data is 


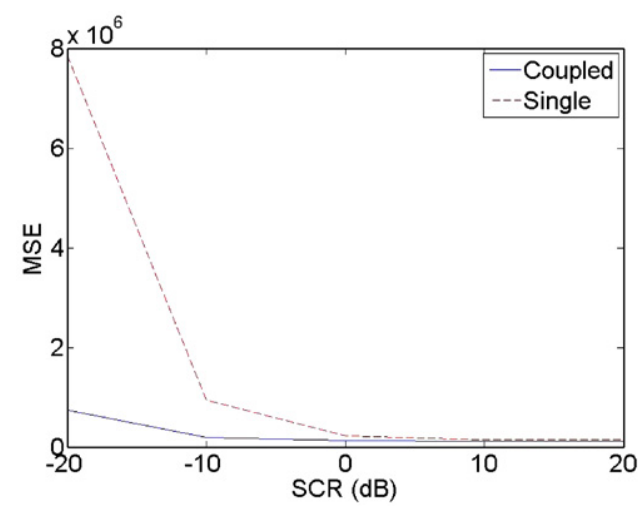

Figure 10. SCR versus MSE for the standard images and coupled images, respectively, $45^{\circ}$ polarized target.
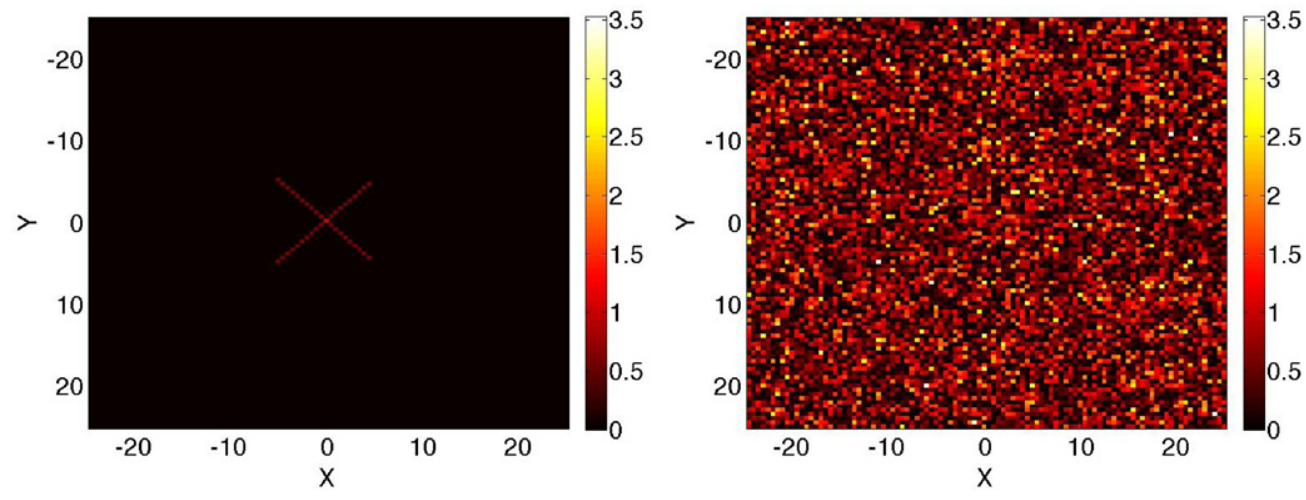

Figure 11. HH component of the target vector and target plus clutter vector, $45^{\circ}$ and $135^{\circ}$ polarized targets.

Table 2. Initial SCR in $\mathrm{dB}$ and final standard versus the coupled image $\mathrm{SCR}$ in $\mathrm{dB}, 45^{\circ}$ polarized target.

\begin{tabular}{rcl}
\hline Scene SCR & Image SCR (standard) & Image SCR (coupled) \\
\hline-20 & 0.1883 & 0.6475 \\
-10 & 0.5955 & 2.0476 \\
0 & 1.8831 & 6.475 \\
10 & 4.3572 & 14.3539 \\
20 & 13.7786 & 45.3912 \\
\hline
\end{tabular}

reduced. Therefore our imaging technique may have the potential to improve images formed from real data.

Example 3: $45^{\circ}$ and $135^{\circ}$ polarized targets Our third example is the case of two targets. The sub-elements of the first target have orientation $\widehat{\boldsymbol{e}}_{T}=[1 / \sqrt{2}, 1 / \sqrt{2}, 0]^{\prime}$ and the second target is made up of sub-elements with orientation $\widehat{\boldsymbol{e}}_{T}=[-1 / \sqrt{2}, 1 / \sqrt{2}, 0]^{\prime}$, for the various $\boldsymbol{x}$ locations. Note the target and target-embedded-in-clutter scenes are displayed in figure 11. In this case, we expect the coupled technique to aid in reconstructing the target as in the previous 

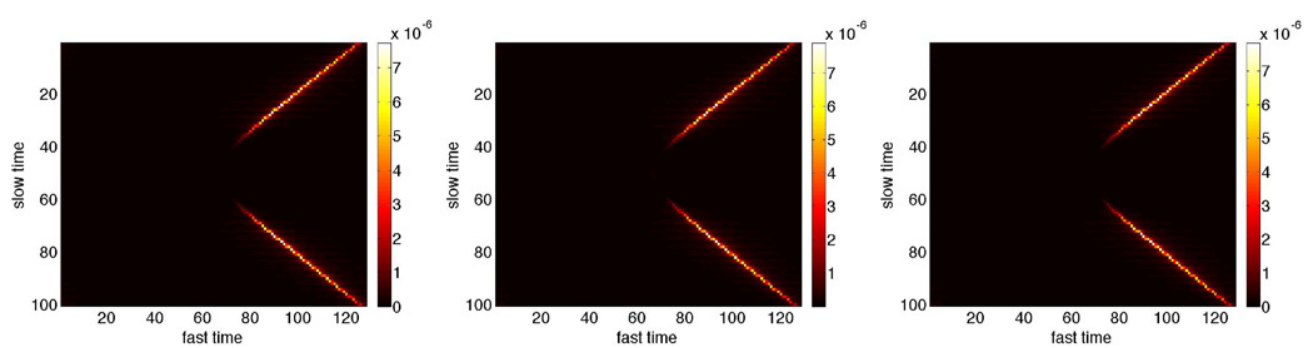

Figure 12. VV, $\mathrm{HH}$ and $\mathrm{HV}$ target only data, $45^{\circ}$ and $135^{\circ}$ polarized target.
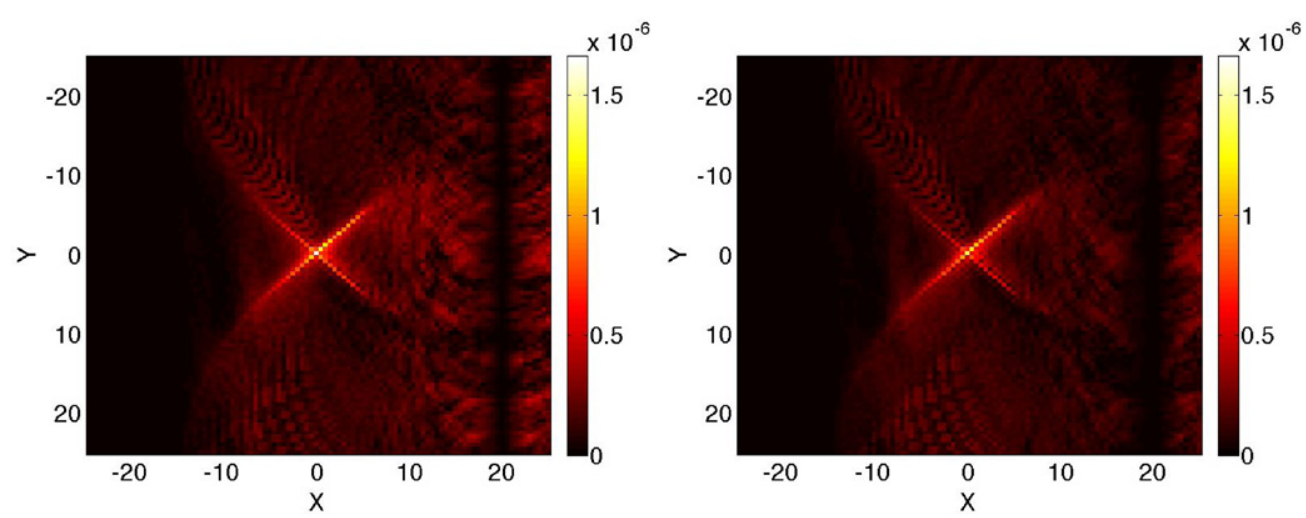

Figure 13. HH image created using the standard (left) and coupled (right) reconstructions, $45^{\circ}$ and $135^{\circ}$ polarized targets.
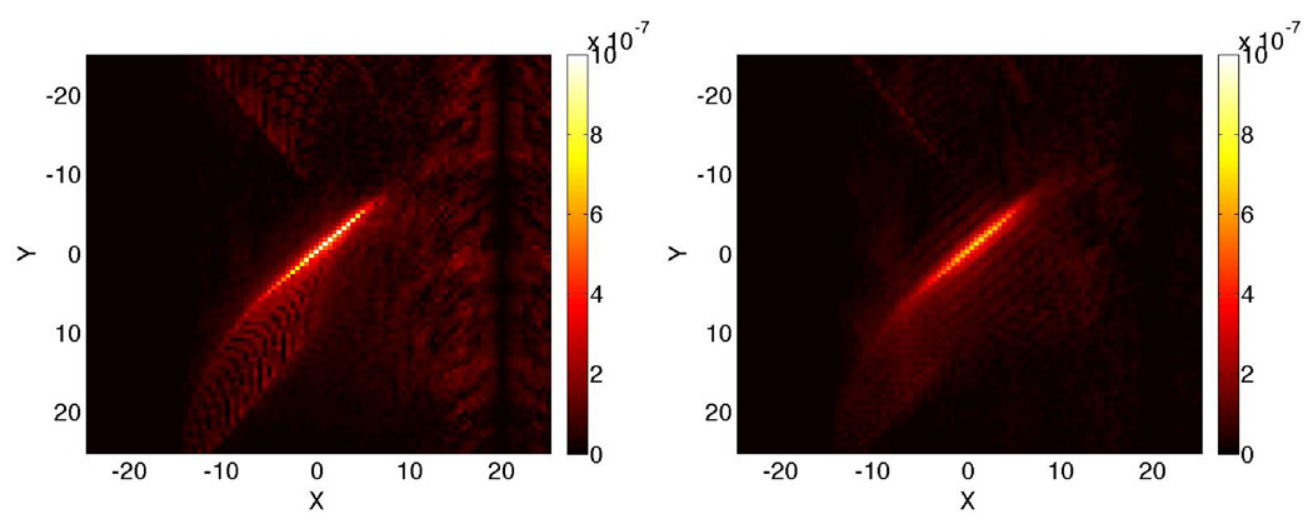

Figure 14. HV image created using the standard (left) and coupled (right) reconstructions, $45^{\circ}$ and $135^{\circ}$ polarized targets.

example. However, the gain in image SCR and decrease in MSE is not as impressive. We note that while the data for all three channels are essentially identical (see figure 12), the HV element of the target scattering vector is negative for the $135^{\circ}$ polarized portion of the target. This leads to a spectral density matrix in the single channel HV case, and also in the coupled case, with elements of smaller magnitude than in the HH and VV cases. This results in the filter matrix $Q$ having elements with decreased magnitude as well. Note we display the $\mathrm{HH}$ and VV components of the image vectors in figures 13 and 14 respectively. Therefore, we see 

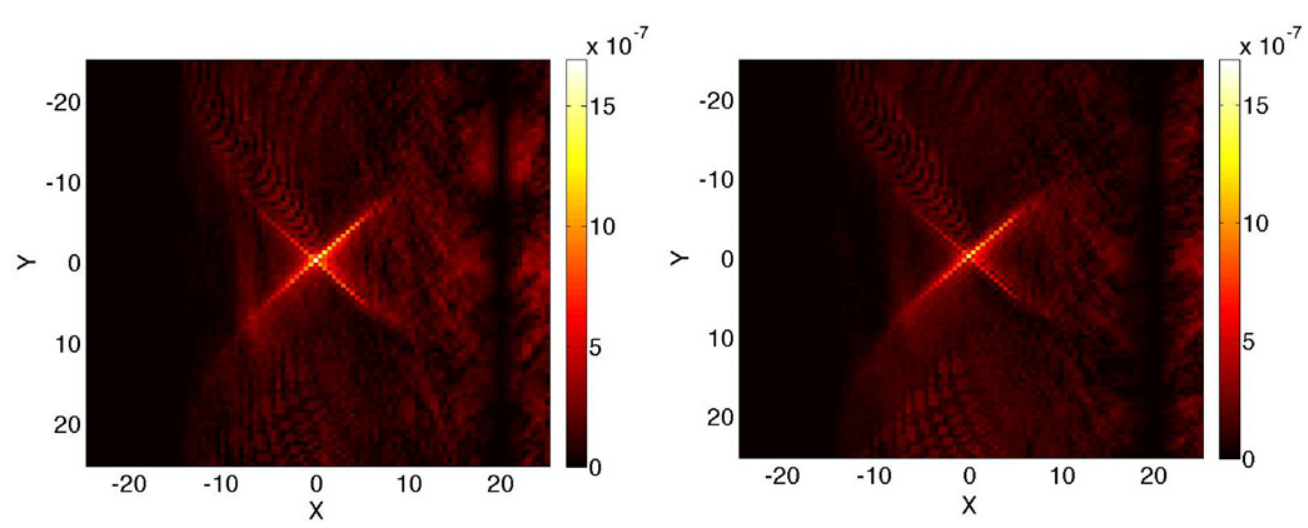

Figure 15. VV image created using the standard (left) and coupled (right) reconstructions, $45^{\circ}$ and $135^{\circ}$ polarized targets.

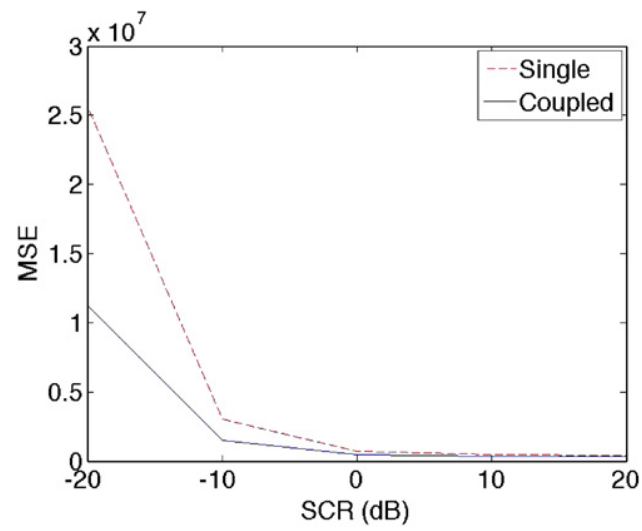

Figure 16. SCR versus MSE for the standard images and coupled images, respectively, $45^{\circ}$ and $135^{\circ}$ polarized targets.

Table 3. Initial SCR in $\mathrm{dB}$ and final standard versus coupled image SCR in $\mathrm{dB}: 45^{\circ}$ and $135^{\circ}$ polarized targets.

\begin{tabular}{ccc}
\hline Scene SCR & Image SCR (standard) & Image SCR (coupled) \\
\hline-20 & 0.1302 & 0.1708 \\
-10 & 0.4118 & 0.5401 \\
0 & 1.3022 & 1.7081 \\
10 & 4.118 & 5.4014 \\
20 & 13.0224 & 17.0808 \\
\hline
\end{tabular}

in figure 14 that both methods fail to reconstruct the $135^{\circ}$ polarized portion of the target in the HV element of the image vector. This change in the filter implies there is less information gained by using the coupled technique in this example. So while we see improvements in figure 16 and in table 3, they are not as significant as in example 2. An adjustment of the filter to compensate for this change in the spectral density matrices would lead to improvements similar to that of the $45^{\circ}$ polarized target case. 


\section{Conclusion}

In this work, we developed a novel polarimetric imaging technique. In particular, our model is useful for imaging targets that display anisotropic scattering behavior. Additionally, we have developed an algorithm that does not rely on the assumption that antenna polarization properties remain constant with respect to the scene of interest. Therefore, our method is potentially useful in a broad variety of scenarios, in particular those where the antenna beam lacks directivity. This technique not only demonstrates how to incorporate statistical knowledge into the imaging scheme, but also demonstrates a way to utilize the additional information polarimetric radar provides.

We have demonstrated that our coupled reconstruction technique improves the image mean-square error and signal-to-clutter ratio. In addition, this method appears to be especially advantageous when the flight path is perpendicular to the target orientation. This work suggests that polarimetric radar may prove useful in improving SAR imaging and target detection. While in this work we have considered an extremely simplified target model, the positive results suggest the need for further work on more complicated target models. To address targets more complicated than curves, the forward model will likely need to be modified, and we leave this for future work.

\section{Acknowledgments}

The authors would like to thank Dr Matthew Ferrara and Dr Richard Albanese for their assistance in formulating this forward problem and inversion scheme. Also we would like to thank Dr Gerald Benitz from MIT Lincoln Laboratory for his suggestion on regularizing the matrix inversion in the numerical simulations. This work was supported by the ATR Center at AFRL $^{6}$ under contract FA8650-08-C-1322, the GAANN fellowship, the National Research Council Postdoctoral Research Associateship program, the Air Force Office of Scientific Research ${ }^{6}$ under contract FA9550-09-1-0013 and the National Science Foundation under grant CCF-08030672.

\section{References}

[1] Allen M R and Hoff L E 1994 Wide-angle wideband SAR matched filter image formation for enhanced detection performance Proc. SPIE 2230 302-14

[2] Bleistein N, Cohen J K and Stockwell J W 2000 The Mathematics of Multidimensional Seismic Inversion (New York: Springer)

[3] Boerner W M, El-Arini M B, Chan C Y and Mastoris P M 1981 Polarization dependence in electromagnetic inverse problems IEEE Trans. Antennas Propag. 29 262-71

[4] Chaney R D, Burl M C and Novak L M 1990 On the performance of polarimetric target detection algorithms IEEE Int. Radar Conf. pp 520-5

[5] Ferro-Famil L, Reigber A, Pottier E and Boerner W M 2003 Scene characterization using subaperture polarimetric SAR data IEEE Trans. Geosci. Remote Sens. 41 2264-76

[6] Chaney R D, Willsky A S and Novak L M 1994 Coherent aspect-dependent SAR image formation Proc. SPIE 2230 256-74

[7] Cheney M and Borden B 2009 Fundamentals of Radar Imaging (Philadelphia, PA: SIAM)

[8] Cloude S R and Pottier E 1996 A review of target decomposition theorems in radar polarimetry IEEE Trans. Geosci. Remote Sens. 34 498-518

6 Consequently, the US Government is authorized to reproduce and distribute reprints for governmental purposes notwithstanding any copyright notation thereon. The views and conclusions contained herein are those of the authors and should not be interpreted as necessarily representing the official policies or endorsements, either expressed or implied, of the Air Force Research Laboratory or the US Government. 
[9] Colton D and Kress R 1983 Integral Equation Methods in Scattering Theory (New York: Wiley)

[10] Papas C H 1965 Theory of Electromagnetic Wave Propagation (New York: McGraw-Hill)

[11] Huynen J R 1970 Phenomenological theory of radar targets PhD Thesis University of Technology, Delft, The Netherlands

[12] Dilsavor R L and Moses R L 1994 Fully-polarimetric GLRTs for detecting scattering centers with unknown amplitude, phase, and tilt angle in terrain clutter Proc. SPIE 2234 14-25

[13] Ertin E and Potter L C 2000 Polarimetric classification of scattering centers using M-ary Bayesian decision rules IEEE Trans. Aerosp. Electron. Syst. 36 738-49

[14] Freeman A and Durden S L 1998 A three-component scattering model for polarimetric SAR data IEEE Trans. Geosci. Remote Sens. 36 963-73

[15] Grigis A and Sjostrand J 1991 Microlocal Analysis for Differential Operators: An Introduction (London Mathematical Society Lecture Note Series vol 196) (Cambridge: Cambridge University Press)

[16] Gustafsson M 2004 Multi-static synthetic aperture radar and inverse scattering Technical Report LUTEDX TEAT-7123

[17] Harrington R F 1961 Time-Harmonic Electromagnetic Fields (New York: McGraw-Hill)

[18] Hajnsek I 2001 Inversion of surface parameters using polarimetric SAR DLR Research Report FB 2001-30 (Oberpfaffenhofen, Germany: DLR)

[19] Huynen J R 1990 The Stokes matrix parameters and their interpretation in terms of physical target properties Proc. SPIE 1317 195-207

[20] Jackson J A and Moses R L 2006 Feature extraction algorithm for 3D scene modeling and visualization using monostatic SAR Proc. SPIE $\mathbf{6 2 3 7}$

[21] Jackson J D 1999 Classical Electrodynamics 3rd edn (New York: Wiley)

[22] King R and Harrison C W 1943 The distribution of current along a symmetrical center-driven antenna Proc. IRE 31 548-67

[23] Kristensson G 1999 Spridningteori med antenntillämpningar (Lund: Studentlitteratur) (in Swedish)

[24] Lee J S and Pottier E 2009 Polarimetric Radar Imaging (Boca Raton, FL: Taylor and Francis)

[25] Lo Monte L 2009 Radio frequency tomography for underground void detection PhD Thesis Department of Electrical Engineering, University of Illinois at Chicago, Chicago, IL

[26] Mott H 1992 Antennas for Radar and Communications: A Polarimetric Approach (New York: Wiley)

[27] Nolan C J and Cheney M 2002 Synthetic aperture inversion Inverse Problems 18 221-36

[28] Novak L M, Burl M C and Irving W W 1993 Optimal polarimetric processing for enhanced target detection IEEE Trans. Aerosp. Electron. Syst. 29 234-44

[29] Novak L M, Sechtin M B and Cardullo M J 1989 Studies of target detection algorithms that use polarimetric radar data IEEE Trans. Aerosp. Electron. Syst. 25 150-65

[30] Peebles P Z 1998 Radar Principles (New York: Wiley)

[31] Ruck G T, Barrick D E, Stuart W D and Krichbaum C K 1970 Radar Cross Section Handbook vol 1 (New York: Plenum)

[32] Tai C T 1952 Electromagnetic back-scattering from cylindrical wires J. Appl. Phys. 23 909-16

[33] Taylor M E 1981 Pseudodifferential Operators (Princeton, NJ: Princeton University Press)

[34] Schou J, Skriver H, Nielsen A A and Conradsen K 2003 CFAR edge detector for polarimetric SAR images IEEE Trans. Geosci. Remote Sens. 41 20-32

[35] Tai C T 1994 Dyadic Green's Functions in Electromagnetic Theory (IEEE Press Series on Electromagnetic Waves) (Piscataway: NJ: IEEE)

[36] Tsang L, Kong J A and Ding K H 2000 Scattering of Electromagnetic Waves: Theories and Applications (New York: Wiley)

[37] van Bladel J 1991 Singular Electromagnetic Fields and Sources (New York: Oxford University Press)

[38] Van de Hulst H C 1981 Light Scattering by Small Particles (New York: Dover)

[39] Van Vleck J H, Bloch F and Hamermesh M 1947 Theory of radar reflection from wires or thin metallic strips J. Appl. Phys. 18 274-94

[40] van Zyl J J 1992 Application of Cloude's target decomposition theorem to polarimetric imaging radar data Proc. SPIE 1748 184-91

[41] Voccola K 2011 Statistical and analytical techniques in synthetic aperture radar imaging PhD Thesis Department of Mathematical Sciences, Rensselaer Polytechnic Institute, Troy, NY

[42] Yazici B, Cheney M and Yarman C E 2006 Synthetic-aperture inversion in the presence of noise and clutter Inverse Problems 22 1705-29 